\title{
Comprehensive molecular, genomic and phenotypic analysis of a major clone of Enterococcus faecalis MLST ST40
}

Melanie Zischka ${ }^{1,2}$, Carsten T Künne ${ }^{3,4}$, Jochen Blom, ${ }^{5,6}$, Dominique Wobser ${ }^{7}$, Türkân Sakınç7, Kerstin Schmidt-Hohagen ${ }^{8}$, P Wojtek Dabrowski ${ }^{9}$, Andreas Nitsche ${ }^{9}$, Johannes Hübner ${ }^{7,10}$, Torsten Hain ${ }^{3}$, Trinad Chakraborty ${ }^{3}$, Burkhard Linke ${ }^{5,6}$, Alexander Goesmann ${ }^{5,6}$, Sonja Voget ${ }^{11}$, Rolf Daniel ${ }^{11}$, Dietmar Schomburg ${ }^{8}$, Rüdiger Hauck ${ }^{12}$, Hafez M Hafez ${ }^{12}$, Petra Tielen ${ }^{13}$, Dieter Jahn ${ }^{13}$, Margrete Solheim ${ }^{14}$, Ewa Sadowy ${ }^{15}$, Jesper Larsen ${ }^{16}$, Lars B Jensen ${ }^{17}$, Patricia Ruiz-Garbajosa ${ }^{18}$, Dianelys Quiñones Pérez ${ }^{19}$, Theresa Mikalsen ${ }^{20}$, Jennifer Bender ${ }^{1}$, Matthias Steglich', Ulrich Nübel ${ }^{1,21}$, Wolfgang Witte ${ }^{1}$ and Guido Werner ${ }^{1 *}$

\begin{abstract}
Background: Enterococcus faecalis is a multifaceted microorganism known to act as a beneficial intestinal commensal bacterium. It is also a dreaded nosocomial pathogen causing life-threatening infections in hospitalised patients. Isolates of a distinct MLST type ST40 represent the most frequent strain type of this species, distributed worldwide and originating from various sources (animal, human, environmental) and different conditions (colonisation/infection). Since enterococci are known to be highly recombinogenic we determined to analyse the microevolution and niche adaptation of this highly distributed clonal type.

Results: We compared a set of 42 ST40 isolates by assessing key molecular determinants, performing whole genome sequencing (WGS) and a number of phenotypic assays including resistance profiling, formation of biofilm and utilisation of carbon sources. We generated the first circular closed reference genome of an $E$. faecalis isolate D32 of animal origin and compared it with the genomes of other reference strains. D32 was used as a template for detailed WGS comparisons of high-quality draft genomes of 14 ST40 isolates. Genomic and phylogenetic analyses suggest a high level of similarity regarding the core genome, also demonstrated by similar carbon utilisation patterns. Distribution of known and putative virulence-associated genes did not differentiate between ST40 strains from a commensal and clinical background or an animal or human source. Further analyses of mobile genetic elements (MGE) revealed genomic diversity owed to: (1) a modularly structured pathogenicity island; (2) a site-specifically integrated and previously unknown genomic island of $138 \mathrm{~kb}$ in two strains putatively involved in exopolysaccharide synthesis; and (3) isolate-specific plasmid and phage patterns. Moreover, we used different cell-biological and animal experiments to compare the isolate D32 with a closely related ST40 endocarditis isolate whose draft genome sequence was also generated. D32 generally showed a greater capacity of adherence to human cell lines and an increased pathogenic potential in various animal models in combination with an even faster growth in vivo (not in vitro).
\end{abstract}

Conclusion: Molecular, genomic and phenotypic analysis of representative isolates of a major clone of E. faecalis MLST ST40 revealed new insights into the microbiology of a commensal bacterium which can turn into a conditional pathogen.

Keywords: Enterococcus faecalis, Whole genome, Esp, Pathogenicity island, Capsule

\footnotetext{
* Correspondence: wernerg@rki.de

'Division of Nosocomial Pathogens and Antibiotic Resistances, Department of Infectious Diseases, Robert Koch Institute, Wernigerode Branch, Burgstr. 37, D-38855 Wernigerode, Germany

Full list of author information is available at the end of the article
}

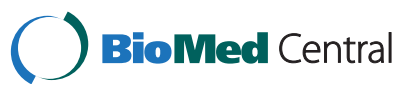

(c) 2015 Zischka et al.; licensee BioMed Central. This is an Open Access article distributed under the terms of the Creative Commons Attribution License (http://creativecommons.org/licenses/by/4.0), which permits unrestricted use, distribution, and reproduction in any medium, provided the original work is properly credited. The Creative Commons Public Domain Dedication waiver (http://creativecommons.org/publicdomain/zero/1.0/) applies to the data made available in this article unless otherwise stated. 


\section{Background}

Enterococci constitute an integral part of the intestinal flora of many invertebrates, birds and mammals including humans. Recent studies on livestock animals have improved our understanding of the role enterococci play as important intestinal colonisers by supporting intestinal, microbial homoeostasis, stimulating immune modulation and thus preventing infections with pathogenic bacteria and viruses [1-3]. Commercial probiotic mixtures of $E n-$ terococcus faecalis are sold with a supposed supportive role in anti-inflammation and prevention of allergic reactions. The genome sequence of one of these probiotic strains called Symbioflor 1 has been released very recently [4]. Enterococci have a supportive activity in food fermentation and preservation, because of their production of secondary metabolites and bacteriocins [5]. On the other hand, enterococci, in particular E. faecalis and E. faecium, are important conditional pathogens. E. faecalis has been attributed to various kinds of infections in humans in hospitals and in the community such as urinary tract infections, bacteraemia and/or endocarditis [6-9]. ${ }^{\mathrm{a}}$ In animals it is a common cause of mastitis in cattle [10], and of urinary tract infections in dogs and cats.

In E. faecalis, a number of 'classical' virulence factors are essential for different kinds of and the course of infections. A cytolysin executes cytolytic, haemolytic and bacteriocinogenic activities capable of lysing prokaryotic and eukaryotic cells [11]. The expression of the twocomponent cytolysin is highly regulated and controlled by quorum-sensing [12]. Relevant genes are arranged in an operon structure which is encoded on a pathogenicity island (PAI) and/or a pheromone response plasmid of the pAD1 type $[13,14]$. The association of cytolysin expression and increased toxicity of $E$. faecalis infections has been shown in various animal models, as well as in outcome-oriented clinical studies [15,16]. Other welldescribed virulence factors such as gelatinase and serine protease have been singly shown to contribute to tissue invasion and translocation and thus to pathogenicity of E. faecalis infections in general [9,17-19]. Corresponding genes gelE-sprE are genetically linked, co-regulated by $f s r$ and co-transcribed [18,20,21].

Examples of $E$. faecalis surface-exposed virulence markers are capsular polysaccharides, glycolipids, surfaceexposed LPxTG-type proteins, such as microbial surface components recognising adhesive matrix molecules (MSCRAMMs), like the collagen-binding protein Ace, pili called Ebp, and aggregation substances [8,22-26]. Surface protein-mediated adherence to host tissues leads to the first steps of enterococcal colonisation and subsequent biofilm formation, inflammation and infection.

The enterococcal surface protein Esp is known to be involved in biofilm production and surface attachment [27-29]. The corresponding esp gene is part of the $E$. faecalis and E. faecium PAI which shows different sizes and compositions in both species [14,30]. The E. faecalis PAI in the reference isolate MMH594 is $153 \mathrm{~kb}$ long and part of the chromosome [14]. In E. faecium only isolates from clinical infections contain the PAI, whereas in $E$. faecalis commensal (human, animal) and environmental strains may also contain it [31,32]. Supposed virulence genes of $E$. faecalis isolates as described above are also distributed among commensal and environmental isolates [33-35]. The exact composition of the E. faecalis PAI varies in different strains. Differences appear in the presence and absence of the six blocks described for the original PAI of MMH594 which are flanked by mobile genetic elements [32]. Parts of the PAI including the 5' encoded aggregation substance may be derived from the integration of plasmid fragments in the PAI. Aggregation substance is also an integral part of various types of $E$. faecalis pheromone plasmids of the pAD1-, pCF10- and pAM373-types $[13,36]$. The aggregation substance is essential in linking donor and recipient cells in pheromoneinduced mating processes but also supports attachment to eukaryotic surfaces and intracellular routes of immune evasion [37,38].

The enterococcal capsule locus (cps) consists of 11 known open reading frames, namely cps $A-K$. There are three known capsule operon polymorphisms: (1) cps type 2, which includes all 11 genes (e.g. V583); (2) cps type 5, which includes all genes except for $\operatorname{cps} F$ (like strains of the CC9); and (3) cps type 1, where only $\operatorname{cps} A$ and $\operatorname{cps} B$ are present (e.g. OG1RF) [24,39].

Horizontal gene transfer (HGT) is supposed to play a key role in shaping enterococcal genomes, bacterial functionality and $E$. faecalis phylogeny [40,41]. This is exemplified by (1) the high number of mobile genetic elements in some sequenced E. faecalis genomes, such as V583 summing up to $>25 \%$ and (2) recent phylogenetic analyses based on whole genome data $[41,42]$. Various integrative and conjugative elements (ICE) are known in Enterococcus, such as the conjugative tetracycline resistance transposons of the Tn916-type and the aforementioned pheromone response plasmids, which allow very efficient gene transfer rates of up to $10 \mathrm{e}-1$ per donor cell. In addition, broad host range plasmids of the Inc18-type are also prevalent among E. faecalis which became prominent when transmitting vanA-type vancomycin resistance from E. faecalis to MRSA [43,44]. ICE can also mobilise other parts of the genomes when transferred as shown recently [45,46]. In E. faecium genome remodelling associated with acquisition and loss of mobile genetic elements has contributed to ecological niche separation with respect to domestic animals and to the evolution of a hospital-associated subpopulation as a cause of infections in immunocompromised humans; within ecological niches and/or distinct clonal 
lineages or complexes the level of genomic variance is minor [47-49].

Data from DNA sequence-based typing of E. faecalis assessed by MLST suggest that a number of prominent clonal complexes which are common among animal and human isolates such as CC2, CC16, and CC40, do not suggest any kind of host specificity and also less abundant clonal lineages were reported from urinary tract infections in humans and chickens [50]. A phylogenetic analysis with respect to ecological separation and host specificity had not been performed for E. faecalis at the time when we initiated our study. Typing by means of MLST of 386 E. faecalis isolates of a European collection revealed that specific types determined by MLST analyses and subsequent eBURST clustering mainly belong to six clonal complexes $(\mathrm{CC} 2, \mathrm{CC} 16, \mathrm{CC} 21, \mathrm{CC} 30, \mathrm{CC} 40$ and CC87) which play a predominant role in the spread of antimicrobial resistance in hospitals and contribute to higher resistance rates in some countries [51]. In order to elucidate further the phylogenetic structure of a prominent MLST type and display possible routes of niche adaptation we focused on the most prevalent clonal type ST40 [39]. We collected and typed 42 ST40 isolates from worldwide and divergent ecological sources by phenotypic and molecular means and characterised a subset of them by whole genome sequence comparisons, functional assays including BIOLOG analyses and animal experiments of colonisation and pathogenicity.

\section{Methods}

\section{Strain collection}

Forty-two isolates of E. faecalis MLST type ST40 were collected from colonisation studies in different animals, people in the community and hospital patients, from different kinds of infections in humans and animals and from food. They originated from different countries and two continents and spanned a range of $>50$ years. A detailed list of the ST40 strains with the corresponding background information is described in Additional file 1: Table S1; summarised information is given in Table 1 . We did not use or receive original clinical samples but only bacterial isolates. Bacteria and plasmids used for reference purposes are given in Table 2.

\section{Antibiotic resistance}

Antibiotic susceptibilities were determined by broth microdilution and according to EUCAST guidelines using clinical breakpoints or epidemiological cut-off values (http://www.eucast.org/clinical_breakpoints/).

\section{Cytolysin/haemolysin and gelatinase assays}

As previously described [45], in vitro $\beta$-haemolytic activity was qualitatively analysed by the use of $\mathrm{MH}$ agar plates containing $5 \%$ human blood in combination with
Table 1 E. faecalis ST40 strain collection used in this study

\begin{tabular}{|c|c|c|c|}
\hline Isolate & Country (City) & Year & Origin \\
\hline UW1833 (U 09508/98)* & $\mathrm{D}$ (Berlin) & 1998 & $\mathrm{H}, \mathrm{U}$ \\
\hline UW5212 & D (Cologne) & 2004 & $\mathrm{H}, \mathrm{U}$ \\
\hline UW5744 & D (Cologne) & 2004 & $\mathrm{H}, \mathrm{U}$ \\
\hline UW6530 & D (Augsburg) & 2006 & $\mathrm{H}, \mathrm{U}$ \\
\hline UW6756 & D (Leezen) & 2006 & $\mathrm{H}, \mathrm{U}$ \\
\hline UW6724 (Ba7514)* & D (Wernigerode) & 2006 & $\mathrm{H}, \mathrm{C}$ \\
\hline UW6727 (Ba7517)* & D (Wernigerode) & 2006 & $\mathrm{H}, \mathrm{C}$ \\
\hline UW7775 & D (Heidelberg) & 2004 & $\mathrm{H}, \mathrm{C}$ \\
\hline UW7776 & D (Heidelberg) & 2004 & $\mathrm{H}, \mathrm{C}$ \\
\hline UW7777 (AK-EF 29)* & D (Heidelberg) & 2004 & $\mathrm{H}, \mathrm{C}$ \\
\hline UW7778 & D (Heidelberg) & 2004 & $\mathrm{H}, \mathrm{C}$ \\
\hline UW7779 (AK-EF 92)* & D (Heidelberg) & 2004 & $\mathrm{H}, \mathrm{C}$ \\
\hline UW2860 $(3803)^{*}$ & D (Gera) & 2000 & $\mathrm{H}, \mathrm{B}$ \\
\hline UW2861 & D (Gera) & 2000 & $\mathrm{H}, \mathrm{B}$ \\
\hline UW4340 & D (Berlin) & 2003 & $\mathrm{H}, \mathrm{B}$ \\
\hline UW4889 & D (Augsburg) & 2004 & $\mathrm{H}, \mathrm{B}$ \\
\hline UW5209 & D (Augsburg) & 2004 & $\mathrm{H}, \mathrm{B}$ \\
\hline UW5213 & D (Augsburg) & 2004 & $\mathrm{H}, \mathrm{B}$ \\
\hline UW5345 & D (Augsburg) & 2004 & $\mathrm{H}, \mathrm{B}$ \\
\hline UW6149 (AB 5093-231) & D (Augsburg) & 2005 & $\mathrm{H}, \mathrm{B}$ \\
\hline UW7800 (ATCC 27275) & Unknown & $\leq 1962$ & Unknown \\
\hline UW7801 (ATCC 27959)* & USA (lowa) & $\leq 1975$ & $A^{1}, M$ \\
\hline UW7729 (LMGT 2333)* & IS (Reijkjavik) & 1990 & $A^{2}, C$ \\
\hline UW7730 (LMGT 3209) & GR (Athens) & $<2004$ & $\mathrm{~F}$ \\
\hline UW7709 $(5)^{*}$ & DK & 1997 & $\mathrm{H}, \mathrm{E}$ \\
\hline UW7710 (6) & DK & $>2000$ & $\mathrm{H}, \mathrm{U}$ \\
\hline UW7742 (D1)* & DK & 2001 & $A^{3}, C$ \\
\hline UW7743 (D27) & DK & 2001 & $A^{3}, C$ \\
\hline UW7744 (D32) & DK & 2001 & $A^{3}, C$ \\
\hline UW7745 (D37) & DK & 2001 & $A^{3}, C$ \\
\hline UW7761 (DQ213/C003-e)* & Cuba (Havana) & 2008 & $\mathrm{H}, \mathrm{B}$ \\
\hline UW7753 (HC 24)* & ESP (Madrid) & 2001 & $\mathrm{H}, \mathrm{B}$ \\
\hline UW7780 $(402 / 96)^{*}$ & PL (Warsaw) & 1996 & $\mathrm{H}, \mathrm{C}$ \\
\hline UW7781 & PL (Warsaw) & 1996 & $\mathrm{H}, \mathrm{PF}$ \\
\hline UW7782 & PL (Warsaw) & 1996 & $\mathrm{H}, \mathrm{U}$ \\
\hline UW7784 & PL (Grajewo) & 1999 & $\mathrm{H}, \mathrm{U}$ \\
\hline UW7785 & PL (Zawiercie) & 2000 & $\mathrm{H}, \mathrm{W}$ \\
\hline UW7787 & PL (Zamość) & 2002 & $\mathrm{H}, \mathrm{CSF}$ \\
\hline UW7788 & PL (Bytom) & 2003 & $\mathrm{H}, \mathrm{B}$ \\
\hline UW7789 & PL (Biała Podlaska) & 2004 & $\mathrm{H}, \mathrm{U}$ \\
\hline UW7790 & PL (Wołomin) & 2007 & $\mathrm{H}, \mathrm{C}$ \\
\hline UW7791 & PL (Maków Maz.) & 2007 & $\mathrm{H}, \mathrm{C}$ \\
\hline
\end{tabular}

E. faecalis strain collection comprises 42 ST40 strains. ${ }^{*}$ draft genomes; ${ }^{\mathbf{\Delta}}$ ST40 complete reference genome; $A$, animal $\left({ }^{1}\right.$ cow ${ }^{2}$ fish, ${ }^{3}$ pig); B, blood culture; $C$, colonizer; CSF, cerebrospinal fluid; $E$, endocarditis; $F$, food (cheese); $H$, human; $M$, bovine mastitis; $P F$, peritoneal fluid; $U$, urine; $W$, wound. 
Table 2 Bacterial strains and plasmids used as references

\begin{tabular}{|c|c|c|c|c|c|}
\hline Strain & MLST & Resistance & Plasmids & Description & References \\
\hline \multicolumn{6}{|l|}{ E. faecalis: } \\
\hline V583 & CC2 (ST6) & VAN, ERY, STR & pTEF1, pTEF2 and pTEF3 & ATCC700802; first sequenced $E$. faecalis genome & [42] \\
\hline MMH594 & CC2 (ST6) & & & Contains the first identified complete PAI & [14] \\
\hline OG1RF & ST1 & RAM, FUS & Plasmid-free & ATCC 47077; mutant of OG1 & [65] \\
\hline \multicolumn{6}{|l|}{ E. faecium: } \\
\hline $64 / 3$ & ST21 & RAM, FUS & None & Commensal isolate; standard recipient & [52] \\
\hline \multicolumn{6}{|c|}{ Staphylococcus aureus: } \\
\hline NCTC8325 & & & & Size marker in PFGE & \\
\hline \multicolumn{6}{|l|}{ Plasmids: } \\
\hline & & & pAD1 & Isolated from E. faecalis OG1::pAD1 & [13] \\
\hline & & TET & pCF10 & Isolated from E. faecalis OG1::pCF10 & [36] \\
\hline & & TET, CMP, ERY, STR & pRE25 & Isolated from E. faecalis RE25::pRE25 & {$[101]$} \\
\hline
\end{tabular}

CC, clonal complex; ST, sequence type; only antibiotic resistances relevant for this study are presented: CMP, chloramphenicol; ERY, erythromycin; FUS, fusidic acid; RAM, rifampicin; STR, streptomycin (high-level); TET, tetracycline; VAN, vancomycin.

1\% glucose (Merck KGaA, Darmstadt, Germany) and 0.03\% L-arginine (Sigma-Aldrich $\mathrm{GmbH}$, St Louis, USA). In vitro gelatinase expression, resulting in hydrolysis of gelatin, was determined using Todd-Hewitt agar plates (OXOID GmbH, Wessel, Germany) containing 3\% gelatin (Becton, Dickinson \& Co., New Jersey, USA) [45].

\section{Isolation of the whole genome DNA}

Genomic DNA was isolated by using column-based methods (Qiagen $\mathrm{GmbH}$, Hilden, Germany). The final DNA concentration was determined using Quant-iT ${ }^{\circledast}$ PicoGreen ${ }^{\circledR}$ dsDNA Quantitation Reagent (Invitrogen Molecular Probes Inc., Paisley, UK) following the instructions of the manufacturer and the quality of the DNA preparation was visually inspected in agarose gels.

\section{Plasmid DNA isolation}

Preparation of plasmid DNA was done by using a phenol/chloroform-based extraction method as described recently [52].

\section{PCR and long template PCR}

Standard PCR reactions were performed using PCR Master mix (Thermo Fischer Scientific Inc., Waltham, USA) in accordance to the manufacturer's instructions. Long template PCR using the Expand Long Template PCR kit (Roche Biochemicals, Mannheim, Germany) was performed to amplify the integration site of a PAI, as well as to analyse its structure according to the reference structure in strain MMH594 and as described recently [45]. All primers used are listed in Additional file 2: Table S2.

\section{Multilocus sequence typing (MLST)}

MLST was performed according to the E. faecalis MLST scheme by amplifying seven housekeeping genes [53].
MLST sequence types (ST) were generated by using the MLST website (http://efaecalis.mlst.net/; last access: 03.03.2015).

\section{PFGE and Southern hybridisation}

SmaI macrorestriction and subsequent PFGE analysis were done as described previously [10]. CHEFF III apparatus (BIO-RAD, Munich, Germany) was used. For plasmid PFGE an S1 nuclease treatment procedure was used as described recently [54]. As an external size standard, SmaIdigested S. aureus NCTC8325 strain was used to calculate the fragment sizes with BioNumerics version 6.0 software (Applied Maths, Sint-Martens-Latem, Belgium).

Southern hybridisations and immunological detection were done with DIG High Prime system kits and CDP-Star detection (Roche Biochemicals, Mannheim, Germany) following the manufacturer's recommendations. In the case of plasmid classification, labelled probes were generated by using DIG-labelled dUTP and primers repCF10-1/2 and repRE25-1/2 for plasmid replicon classification (Additional file 2: Table S2).

\section{Sequencing by Sanger ABI Big Dye technology}

PCR products were sequenced by a cycle sequencing approach according to the recommendations of Applied Biosystems (Darmstadt, Germany). Sequencing amplicons were determined at the central sequencing facility of the RKI. Sanger reads were analysed by Lasergene 8 (DNASTAR, Madison, USA) or DS Gene software packages (Accelrys, Inc., San Diego, USA).

\section{Roche/454 FLX genomic pyrosequencing}

Fifteen representative isolates were selected for de novo sequencing by Roche/GS-FLX 454 technology. To generate a completely closed, circular ST40 reference genome 
the porcine strain D32 was used for combined long and short template paired end library 454 sequencing at MWG Biotech (Ebersberg, Germany) as described briefly in [55]. Genomic contigs were generated and assembled by using the Newbler assembler software (Roche Diagnostics, Basel, Switzerland).

\section{Illumina/Solexa sequencing and hybrid assembly}

To improve the accuracy of the sequencing data, genomes of the remaining 14 E. faecalis strains (without D32 ([55]) were additionally sequenced by using v2 chemistry and Illumina's Genome Analyzer IIx (Nextera DNA Sample Preparation Kit, Illumina, San Diego, USA). De novo sequences, generated by both Roche/454 FLX pyrosequencing and Illumina/Solexa sequencing, were assembled in a single approach (hybrid assembly) by using Mira assembler software (Sourceforge/Dice Holdings, Inc., N.Y., USA).

\section{Genomic comparisons and phylogenetic analyses}

Generating a reference genome of isolate D32 was described previously [55]. Annotation was done with RAST and GenDB [55]. Circular maps visualising the E. faecalis ST40 genome comparison against the D32 reference genome were generated by BRIG [56]. Alignments for the phylogenetic tree were calculated with Mugsy [57] and only the aligned regions present in all analysed strains were extracted ('core genome'). These regions were concatenated and positions with gaps removed [58]. The resulting core alignment was used to infer a Maximum Likelihood tree with RAxML [59]. The GTRGAMMA model for nucleotide substitution and rate heterogeneity was utilised, and bootstrap support values of 1000 replicates were shown at the nodes. Additional phylogenetic analyses of the 15 E. faecalis ST40 isolates were performed as follows: Solexa single reads were mapped onto the reference sequence of $E$. faecalis D32 by utilising a pipeline based on bwa version: 0.7.3a (http://sourceforge.net/projects/bio-bwa/files/) for mapping, SAM tools 0.1.18 (http://samtools.sourceforge.net/) for SAM/BAM file handling and VarScan v2.3 (http:// varscan.sourceforge.net/index.html) for variant calling. In this way consensus sequences aligned to the reference sequence were generated and all variable sites were extracted. To exclude SNPs resulting from recombination the distances between SNP positions were analysed to find pairs within a certain range (exclusion distance $\leq 300 \mathrm{bp}$ ). Subsequently, a total of 1481 variable positions were used for tree calculations by the PhyML algorithm with a bootstrap of 1000. The genome sequence of E. faecalis D32 is deposited at GenBank numbers NC_018221-018223. All other scaffolded E. faecalis genome sequences are deposited at JGI's IMG/ER website (https://img.jgi.doe.gov/ cgi-bin/er/main.cgi) under the accession numbers 22305 22317 (see also Additional file 1: Table S1).

\section{Biolog phenotyping microarrays}

Biolog Phenotyping Microarrays (PM01 and PM02A MicroPlate $^{\mathrm{rm}}$; BIOLOG, Hayward, USA) were used to analyse the metabolic phenotype with focus on utilisation of different carbon sources under aerobic conditions. Briefly, bacteria were incubated on fresh $\mathrm{MH}$ agar plates (OXOID GmbH, Wessel, Germany) at $37^{\circ} \mathrm{C}$ for $24 \mathrm{~h}$. With a sterile swab, bacteria were transferred into a sterile tube containing $10 \mathrm{~mL}$ IF-0a medium (1.2x) (BIOLOG), and cell suspension was adjusted to $81 \%$ transmittance by using the Biolog turbidimeter (BIOLOG). In line with the manufacturer's recommendations, $20 \mathrm{~mL}$ of the IF-0a Base (1.2x) was mixed with 0.24 mL Redox Dye Mix D (100x) (both BIOLOG) and $2.0 \mathrm{~mL}$ of a $12 \mathrm{x}$ additive solution, containing $24 \mathrm{mM} \mathrm{MgCl} 2 \times 6 \mathrm{H}_{2} \mathrm{O}$ (CARL ROTH GmbH \& Co. KG, Karlsruhe, Germany) and $12 \mathrm{mM} \mathrm{CaCl}_{2} \times 2$ $\mathrm{H}_{2} \mathrm{O}$ (CARL ROTH GmbH \& Co. KG, Karlsruhe, Germany). Finally, $1.76 \mathrm{~mL}$ of cell suspension was added. $100 \mu \mathrm{L}$ of the final suspension was added to each well of the 96-well Biolog PM01 and PM02A plates. The panels were placed in the OmniLog instrument (BIOLOG) and incubated at $37^{\circ} \mathrm{C}$ for $72 \mathrm{~h}$, whereby the utilisation of the carbon sources was measured spectrophotometrically and recorded every $15 \mathrm{~min}$ over the whole incubation period. All assays were repeated for at least three times on different days. Data sets were evaluated by the OmniLog Kinetic and Parametric analysis software version 2005 (BIOLOG Life Science Institute, Bremen, Germany). The area under the kinetic curve (area values) was used to compare the utilisation of different carbon sources between the strains. Calculation of the arithmetic averages with the corresponding standard deviations was done with Microsoft Excel. Colour codes, used in Additional file 2: Table S6a and $b$, were chosen according to Gripp and colleagues [60].

\section{Growth kinetics}

To determine the bacterial growth rates, overnight cultures were diluted 1:50 in TS broth and grown at $37^{\circ} \mathrm{C}$ with shaking. Optical density at $600 \mathrm{~nm}$ was measured at different time points and the corresponding CFU calculated by plating serial dilutions of the cultures onto LB agar plates in duplicate.

\section{Biofilm plate assay}

The ability of selected E. faecalis strains to produce biofilm on flat bottom polystyrene microtiter plates (Greiner Bio-one, Germany and Corning Inc., New York, USA) was tested by following the methodology previously described [45]. Biofilm plate assay for each of the strains was done in triplicate and repeated twice. Statistical comparisons were done by unpaired two-tailed t-tests using a GraphPad Prism 5.01 software package (GraphPad Software, Inc., La Jolla, USA). A $P$-value of $<0.05$ was considered statistically significant. 


\section{Adherence assays}

Adherence to human epithelial colorectal adenocarcinoma Caco-2 cells was investigated using a protocol previously described [22,23] with slight modifications. Caco-2 cells between the 15th and 25th passages were cultivated in 24-well plates to a density of $1 \times 10^{5}$ cells/ well for 13 to 15 days (confluent monolayer). The monolayers were incubated with a bacterial cell to epithelial cell ratio of 100:1, as well as 1000:1 for $2 \mathrm{~h}$. After infection of the monolayer, epithelial cells were washed five times with phosphate saline buffer (PBS, Biochrom AG) and lysed with $0.25 \%$ Triton-X100 (Sigma) at $37^{\circ} \mathrm{C}$ for 15 minutes. To determine quantitatively the number of attached bacterial cells, lysates were diluted in TSB (tryptic soy broth) and plated onto TSA plates. Statistical comparisons of cfu numbers were done by unpaired two-tailed t-tests using the GraphPad Prism 5.01 software package (GraphPad Software, Inc., La Jolla, USA).

\section{Animal models}

Different animal models were used for a comparative assessment of the pathogenic potential of the selected enterococcal strains D32 and UW7709. Also, E. faecalis strains V583 and OG1RF, as well as E. faecium 64/3, served as controls.

\section{Assay of G. mellonella infection}

The insect larva G. mellonella is an alternative model for studying bacteria-host interactions, showing a complex immune reaction consisting of both cellular and humoral responses. According to previously described protocols $[61,62]$, assays were done with some modifications. Some $100 \mu \mathrm{L}$ of TSB overnight culture was added to $5 \mathrm{~mL}$ of fresh TSB medium (Becton, Dickinson \& Co., Heidelberg, Germany) and cultured at $37^{\circ} \mathrm{C}$ for $3 \mathrm{~h}$. After centrifugation for $5 \mathrm{~min}$ at $8,000 \mathrm{rpm}$, cell pellets were resuspended in $1 \mathrm{~mL}$ sterile PBS. Cell concentration was photometrically measured and the cell density of the inoculum was adjusted to $10^{7}$ cells $/ 500 \mu \mathrm{L}$. Groups of $15 \mathrm{G}$. mellonella larvae (www.reptilienkosmos.de) with a weight of about $200 \mathrm{mg}$ were separated. Then, $5 \mu \mathrm{L}$ of the bacterial inoculum was microinjected at the base of the last proleg, corresponding to an infective dose of $10^{5} \mathrm{CFU} /$ larvae. A control group of larvae was infected with PBS only. The real infective dose was determined by serial dilution, plated on PBS agar plates. Groups of infected larvae were kept per Petri dish at $37^{\circ} \mathrm{C}$ and the number of dead larvae was counted after 18, 24, 42, 48, 66 and $70 \mathrm{~h}$. This approach was repeated at least three times for each of the selected strains. Using the nominal values of survival and death, the diagram, presenting the death rates, was calculated by Kaplan-Meier plot method in GraphPad Prism 5.01 software version (GraphPad Software, Inc., La Jolla, USA). Statistical significance $(\mathrm{p}<0.05)$ was determined by the Log-rank (Mantel-Cox) test.

\section{Murine bacteraemia model}

The murine bacteraemia model was used to evaluate the pathogenic potential of the selected E. faecalis strains D32 and UW7709 by analysis of bacterial growth in blood and murine organs (liver, kidney and spleen) following a methodology described previously [23,63]. In brief, eight female 6-8-week-old BALB/c mice (Charles River Laboratories Internations, Inc., Wilmington, USA) were inoculated intravenously in the tail vein with $10^{8}$ and $5 \times 10^{8} \mathrm{CFU}$ respectively and bacterial growth was analysed after $48 \mathrm{~h}$ post-infection. Statistical comparisons were done by Mann-Whitney tests (non-parametric data) using the GraphPad Prism 5.01 software package (GraphPad Software, Inc., La Jolla, USA).

Accession numbers. The full genome sequence of $\mathrm{E}$. faecalis D32 is deposited at GenBank numbers NC018221018223. All other scaffolded E. faecalis genome sequences are deposited at JGI's IMG/ER website (https://img.jgi.doe. gov/cgi-bin/er/main.cgi) under the accession numbers 22305 - 22317 (see also Additional file 1: Table S1).

\section{Results}

\section{Pre-characterisation of the ST40 strain collection}

The ST40 strains were compared for specific phenotypic and genotypic characteristics in order to select representative isolates for genome sequencing. Strains were typed by SmaI macrorestriction in PFGE and sub-clusters of closely related strains were identified which were grouped independently of their geographical and temporal origin or clinical/non-clinical context (Figure 1). Distribution of phenotypic antibiotic susceptibilities was completed by determination of the corresponding genes by PCR (Table 3; Additional file 1: Table S1). Results were mainly congruent. Two discrepancies were detected; one isolate was aadE-positive but not streptomycin-high-level resistant and another isolate was aac6'-aph2"-negative but gentamicinresistant. Presence and expression of putative virulenceassociated genes encoded within the $E$. faecalis PAI and/or on the chromosome were investigated by PCR and partly confirmed by phenotypic in vitro assays (Table 4). The PAIassociated aggregation substance asc-10 gene was found in $16.7 \%$ of the isolates. Prevalence of the cytolysin (cyl) operon in $33.3 \%$ of the strains was associated with the evidence of $\beta$-haemolysis in vitro. The enterococcal surface protein gene esp was detected in $78.6 \%$ of the ST40 strains. All isolates harboured the gelE (gelatinase) and $f s r$ (major accessory gene regulator) genes and showed in vitro gelatinase expression (Table 4; Additional file 1: Table S1).

The enterococcal capsule locus (cps) consists of 11 known open reading frames, namely $c p s A-K$. Capsule locus type 1 ( $c p s A-c p s B)$ was verified by PCR for all ST40 isolates.

We also tested the ability of the ST40 strains to form biofilms on polystyrene plates. Biofilm formation was 


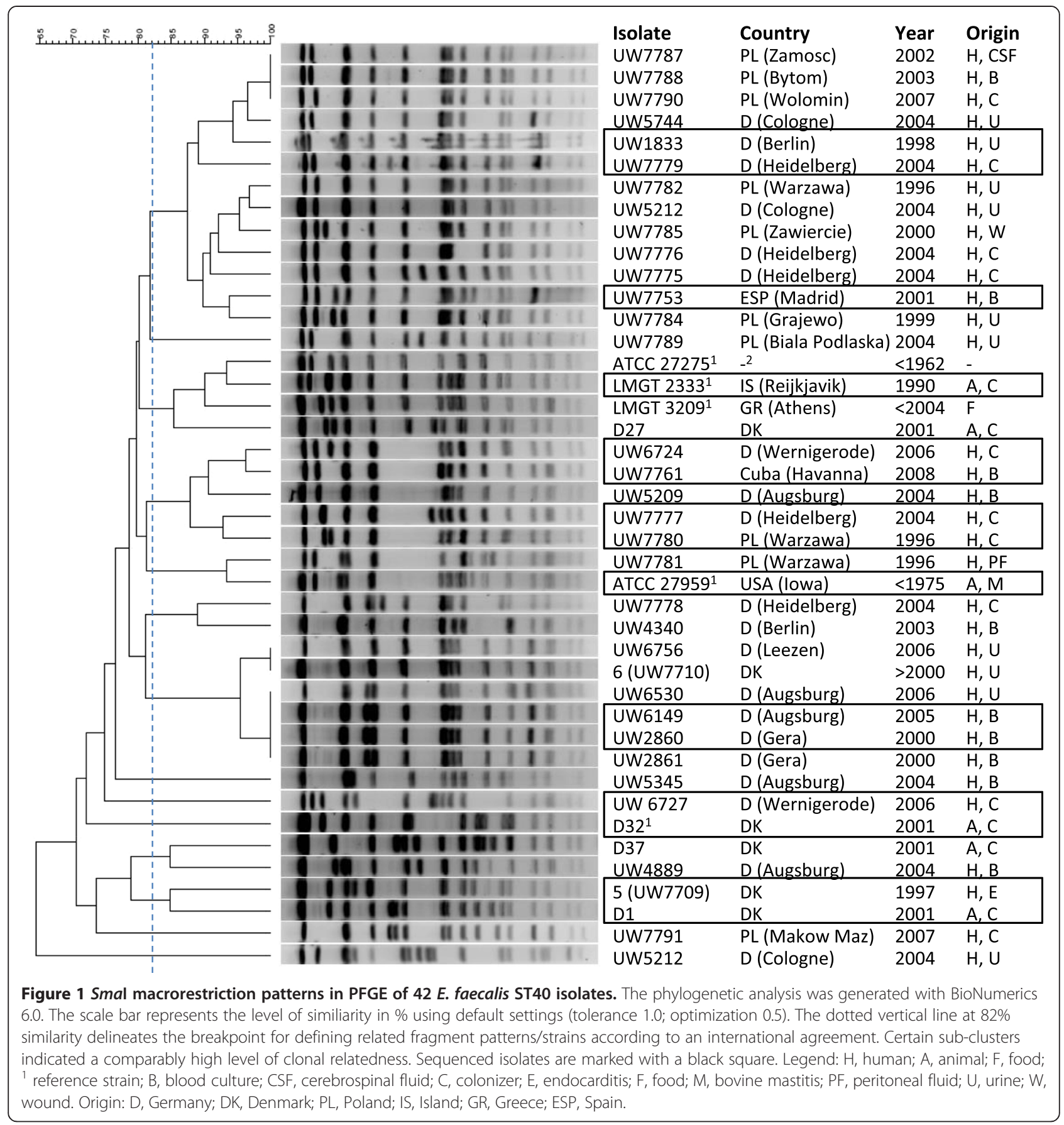

Table 3 Distribution of antibiotic resistances in ST40 E. faecalis isolates

\begin{tabular}{lll}
\hline Antibiotic resistance & Phenotype [\%] & Resistance gene \\
\hline Vancomycin (VanA-type) & $2.4(1 / 42)$ & vanA \\
Erythromycin & $21.4(9 / 42)$ & erm $(\mathrm{B})$ \\
Tetracycline & $81.0(34 / 42)$ & tet(M) \\
Streptomycin (high-level) & $31.0(13 / 42)$ & aadE \\
Gentamicin (high-level) & $21.4(9 / 42)$ & aac6'-aph2" \\
\hline
\end{tabular}

Broth microdilution assay was used to determine phenotypic antibiotic susceptibilities. Resistance genes were determined by PCR. 
Table 4 Presence and expression of putative virulence factors in ST40 E. faecalis isolates

\begin{tabular}{llll}
\hline Putative virulence factor & Putative function & \% of positive isolates & Comment \\
\hline Pathogenicity island & & $16.7(7 / 42)$ & Expression of $\beta$-hemolysis in vitro \\
asc-10 & Aggregation substance & $33.3(14 / 42)$ & $78.6(33 / 42)$ \\
cylM & Cytolysin subunit modifier & $4.8(2 / 42)$ & $0(0 / 42)$ \\
esp & Enterococcal surface protein & \\
xyl kinase & Xylose kinase & $100(42 / 42)$ & Expression of gelatinase in vitro \\
gls24-like & General stress protein & $100(42 / 42)$ & \\
Other & & $100(42 / 42)$ & \\
gelE & Gelatinase/coccolysin & Accessory gene regulator B & Capsular polysaccharide
\end{tabular}

Presence of putative virulence-associated genes (in italics) was examined by PCR. Numbers in bold represent percentage of positive tested isolates, calculated from the number of the positive genotypes versus the whole strain collection (in parentheses).

independent of the presence of putative biofilmenhancing genes like $e s p$ and $a s c-10$, and was also inhomogeneous between closely related strains (Additional file 3: Figure S1).

Results of plasmid isolation in combination with S1 nuclease PFGE indicated diversity in plasmid content, varying in size and quantity (none to two plasmids) and plasmid rep type (see "Comparative analysis of the $E$. faecalis ST40 genomes" and Additional file 4: Figure S2).

\section{Genome sequencing}

On the basis of previous characterisations, a subset of 15 strains (Figure 1), representing the diversity of the ST40 collection and some pairs of related isolates, was sequenced de novo by Roche GS FLX 454 technology. Genomic contigs were generated and assembled by using the Newbler assembler software (Additional file 2: Table S3). Although in principle suitable for de novo genome assemblies, classical 454 sequencing revealed only two strains (D32 and UW7709) with fewer than 100 genomic contigs. The calculated genome size varied from 2.8 to $3.3 \mathrm{Mbp}$. For the majority of the genomes coverage was unsatisfactory $(<20 \mathrm{x})$. To improve the accuracy and overall quality of the sequencing data, a subsequent workflow was pursued including: (1) establishing a template for genomic mapping within ST40 by generating a completely closed chromosome of a reference isolate using long paired end $8 \mathrm{kbp}$ libraries and 454 sequencing as well as classical Sanger sequencing to correct for InDel errors; and (2) additional sequencing of the other 14 E. faecalis strains by Solexa technology (see also Methods and ff).

\section{Generating a reference genome of $E$. faecalis D32}

Key parameters of the sequencing, assembly and annotation strategy were described recently as well as standard information regarding numbers of ribosomal genes, coding sequences, etc. [55]. Additionally to what we have elucidated in this previous announcement, subsequently performed S1 nuclease PFGE analyses revealed a single ca. $75 \mathrm{~kb}$ plasmid in strain D32. Regarding the two supposedly assembled plasmid contigs of 62 and $13 \mathrm{~kb}$ and the fact that the former plasmid EFD32pB did not show any homology with a replicase gene, we postulated an assembly error and suggested the two contigs merge into one single plasmid sequence demonstrating rep1 type.

We used a Venn diagram presentation generated by the web application EDGAR to illustrate homologies and differences between the finished and publicly available $E$. faecalis genomes. It illustrated a common gene pool of 2173 CDS, present in all of the finished E. faecalis genomes (Figure 2). This analysis also revealed that the number of unique CDS of the clinical strain V583 was approximately twice that of the counts of the commensal isolates 62 and our ST40 isolate D32 as well as the probiotic Symbioflor 1 strain. As a derivate of the commensal isolate, the OG1RF strain carried the minimal number of 140 unique CDS. The commensal strains 62 and D32 both shared significantly more CDS with the V583 chromosome, whereas the Symbioflor 1 and OG1RF chromosomes overlapped less with the V583 core genome.

\section{Comparative analysis of the $E$. faecalis ST40 genomes}

De novo sequences, generated by both 454 pyrosequencing and Illumina/Solexa sequencing, were hybrid assembled. This combined sequencing and assembly approach resulted in satisfactory analysis parameters since it improved overall coverage and read length, slightly increased the genome size $(2.928-3.33 \mathrm{Mbp})$ and reduced the number of genomic contigs $(<100$; Table 5$)$. Mapping of the 14 ST40 draft genomes against the D32 reference genome suggested a high level of genomic 


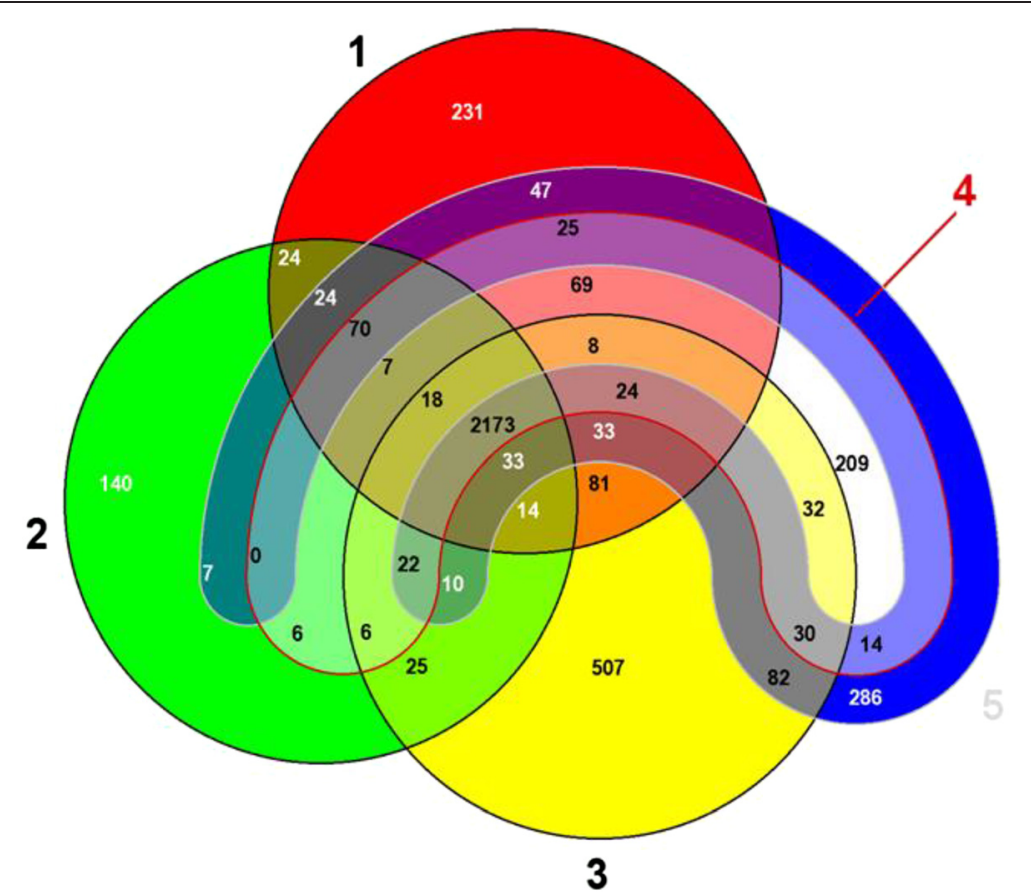

Figure 2 Comparative analysis of the finished and publicly available $\boldsymbol{E}$. faecalis genomes. EDGAR generated Venn diagram facilitates visualizing core and strain-specific ("unique") genes. This comparative analysis only exploits CDS of the chromosomes without considering of plasmid genes, whereby all strains shared 2173 CDS; 1, E. faecalis strain 62 (CP002491); 2, E. faecalis strain OG1RF (CP002621); 3, E. faecalis V583 (NC_004668); 4, E. faecalis probiotic strain Symbioflor 1 Clone DSM 16431 (NC_019770); 5, E. faecalis strain D32 (CP003726).

similarity irrespective of the geographical, host, temporal or clinical/non-clinical origin of the isolates (Figure 3). Differences between the strains were minor and corresponded to (1) a different composition of the E. faecalis PAI, (2) differences in phage content and (3) a putative genomic island first described in D32 which is absent in all other ST40 strains except for another animal isolate, UW7729. This genomic island was located at a putative hot spot for integration in the E. faecalis chromosome, since strain V583 contained the $\operatorname{vanB}$ operon at this site

Table 5 Quality report of hybrid assemblies considering of 454 and Solexa sequencing data

\begin{tabular}{lllllll}
\hline Isolate & No. of assembled reads & Coverage (454) [n-fold] & Coverage(Solexa) [n-fold] & No. scaffolds & Genome size [bp] & GC content [\%] \\
\hline UW6149 & $5,060,490$ & 12.78 & 166.36 & 72 & $3,011,563$ & 37.46 \\
UW2860 & $5,318,329$ & 14.68 & 176.92 & 74 & $3,003,615$ & 37.49 \\
UW6724 & $1,232,615$ & 9.91 & 39.94 & 38 & $3,085,224$ & 37.33 \\
UW7761 & $2,025,698$ & 12.71 & 81.94 & 26 & $2,939,826$ & 37.53 \\
UW7777 & $1,504,959$ & 24.05 & 45.28 & 24 & $2,999,678$ & 37.43 \\
UW7780 & $1,108,686$ & 10.91 & 37.28 & 38 & $3,115,640$ & 37.31 \\
UW7753 & $1,424,574$ & 11.14 & 39.48 & 67 & $3,119,011$ & 37.09 \\
UW1833 & $3,127,985$ & 100.92 & 66 & $3,243,986$ & 37.05 \\
UW7779 & $1,412,336$ & 14.89 & 37.1 & 45 & $3,070,536$ & 37.20 \\
UW7729 & 972,823 & 18.92 & 28.64 & 75 & $3,094,285$ & 37.19 \\
UW7801 & $1,478,988$ & 9.01 & 56.72 & 26 & $2,933,628$ & 37.52 \\
UW6727 & $1,442,566$ & 10.79 & 45.58 & 34 & $3,330,760$ & 36.91 \\
UW7709 & $2,403,224$ & 12.5 & 90.18 & 36 & $2,928,951$ & 37.30 \\
UW7742 & $3,271,151$ & 12.98 & 101.23 & 64 & $3,054,136$ & 37.17 \\
\hline
\end{tabular}

Genomes of 14 selected ST40 isolates were sequenced by using Solexa technology. Reads of 454 and Solexa sequencing were hybrid assembled by using Mira assembler software and resulted in an increased coverage in combination with a reduction of the number of large contigs. 


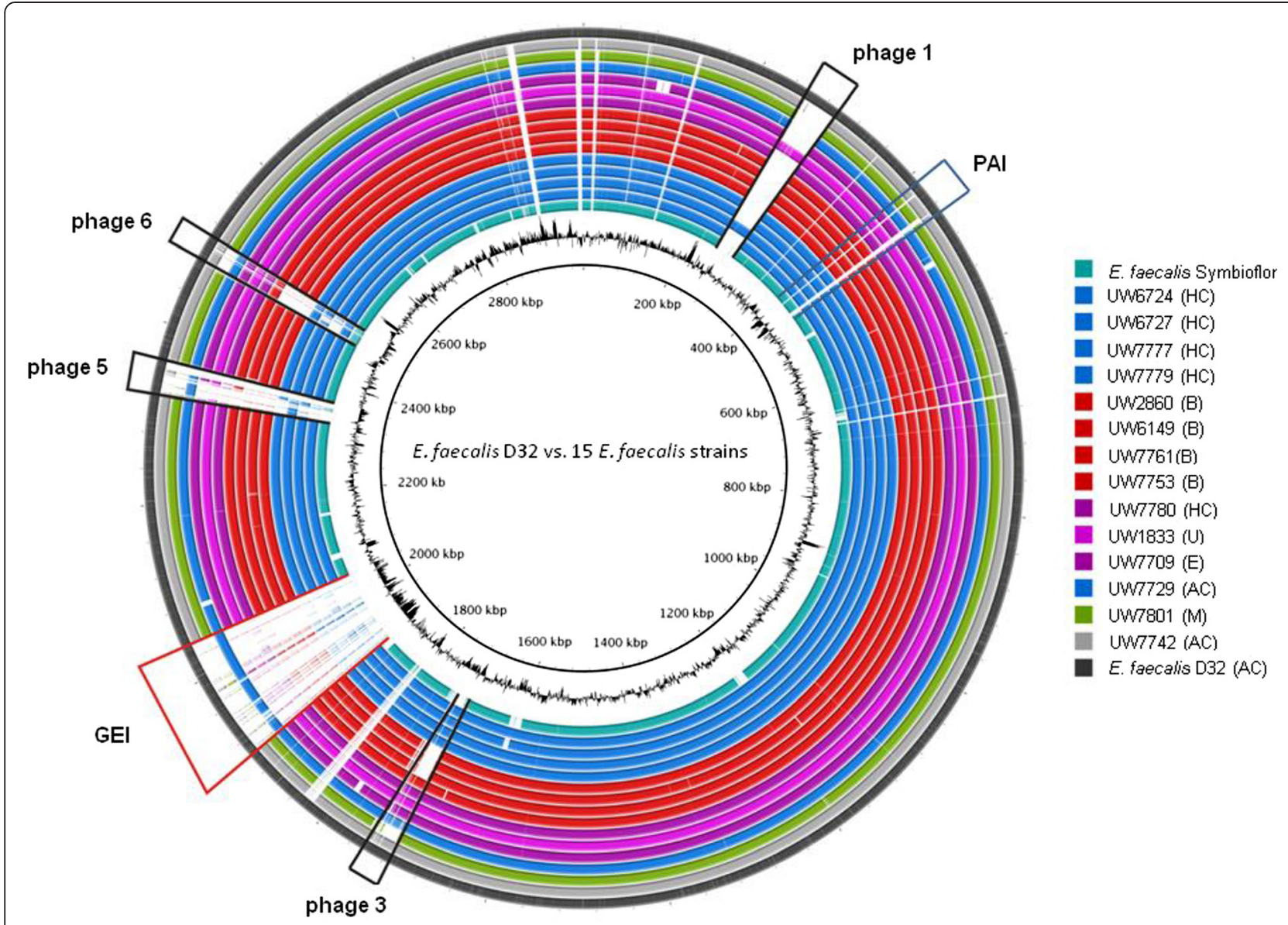

Figure 3 E. faecalis ST40 genome comparison against the D32 reference genome. Generated by BRIG (http://brig.sourceforge.net/ [last access 16.07.2014] [56]), the circular map illustrates the whole genome comparison of D32 against the other 14 sequenced ST40 isolates and the probiotic isolate Symbioflor 1 Clone DSM 16431. The outer cycle (dark grey) represents the complete genome of the reference strain D32. The shade of color is geared to similarities in origin of the strains (green: isolate from bovine mastitis; blue: animal and human commensals; violet: isolates from human infections; red: human blood culture isolates; turquoise: strain Symbiolfor 1). The inner cycle illustrates the GC content of D32. Location of the PAl is illustrated by a blue colored box, while the red box indicates the presence of an uncharacterized and large genomic island (Gl; 138 kb). Additionally, black labels highlighted four identified prophages of D32; A, animal; B, blood culture; C, colonizer; E, endocarditis; $H$, human; $M$, bovine mastitis; $U$, urine.

whereas OG1RF harboured the myo-inositol operon (which D32 lacks).

A phylogenetic tree resulting from an alignment of concatenated sequences, present in all analysed strains and after elimination of existing gaps, is shown in Figure 4. It revealed a very high level of genomic similarity of unrelated ST40 strains, despite their diverse origins and the time interval from $<1960$ to 2009. Of note, strains of a similar origin were not arranged in the same clusters. When we focused on the ST40 isolates, the coregenome based phylogenetic tree also showed an exceptional position of D32 in relation to the other sequenced ST40 isolates and furthermore its close relationship with the other Danish porcine isolate UW7742. As expected, the completely closed and publicly available $E$. faecalis genomes branch separately, supporting their assignment to different sequence types and clonal complexes based on
MLST. In order to confirm the relationship between the 15 E. faecalis ST40 isolates with respect to their core genome, additional phylogenetic analyses were performed by mapping Solexa single reads of 14 isolates against the $E$. faecalis D32 reference sequence using a mapping pipeline based on bwa. As enterococci frequently undergo chromosomal rearrangements, we first excluded SNPs which were owed to recent recombination events. This yielded a total of 1481 variable positions (SNPs), which in turn served as the basis for tree reconstruction by the PhyML algorithm. The generated tree in Additional file 5: Figure S3 revealed a highly similar structure to the previous one (Figure 4) despite the different input data supporting the reliability of both approaches. Both trees revealed exactly identical subclusters of related strains. In Additional file 5: Figure S3 the separate clustering of the two pig commensal strains D1 and D32 from Denmark is highly visible and supported 


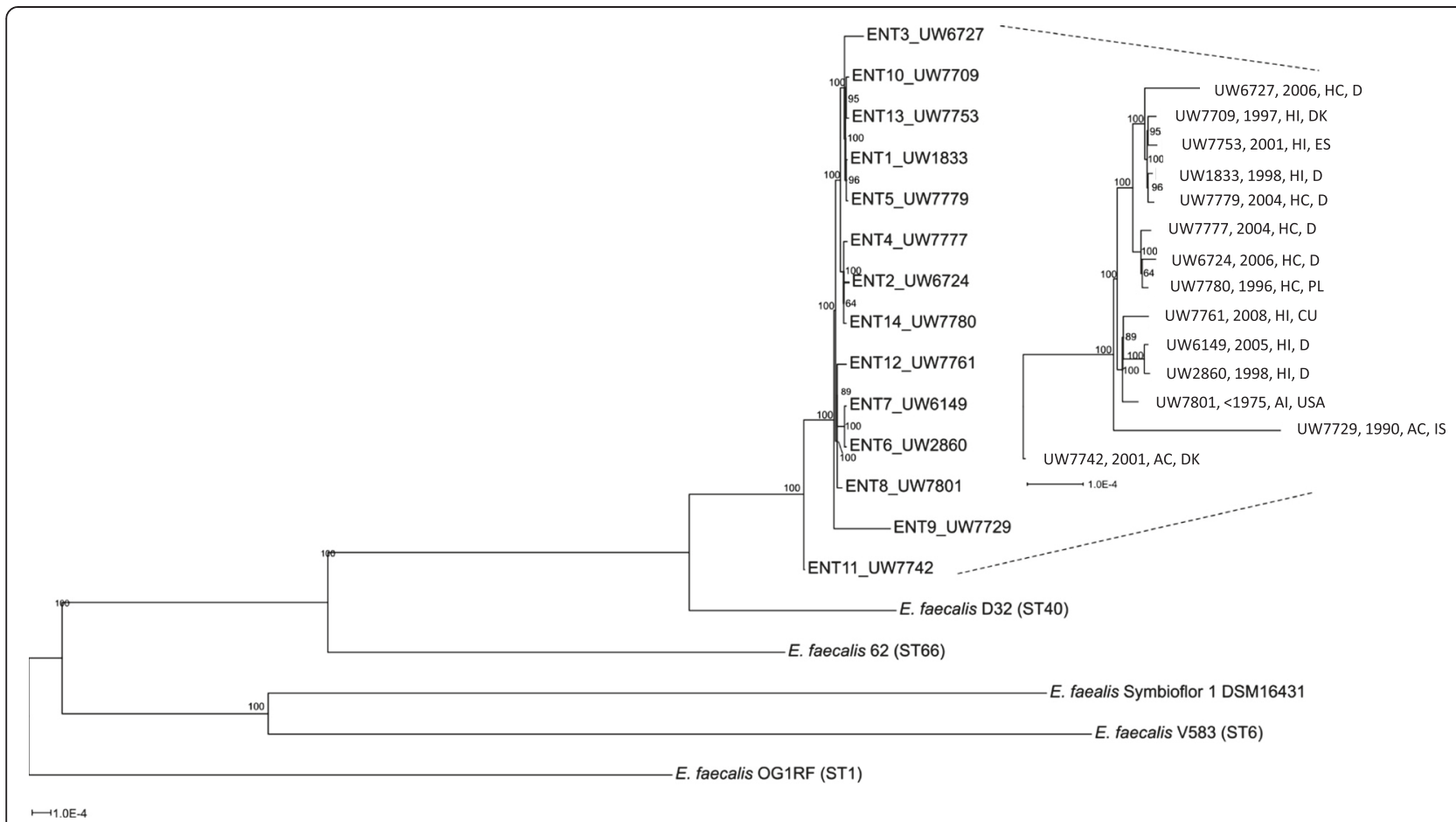

Figure 4 Phylogenetic relationship among selected $E$. faecalis strains based on whole genome alignments. The alignment was calculated with Mugsy (http://mugsy.sourceforge.net/ [last access 16.07.2014] [57]) and only aligned regions present in all analyzed strains were extracted. These regions were concatenated and positions with gaps removed. The resulting core alignment was used to infer a Maximum Likelihood tree with RAXML. The GTRGAMMA model for nucleotide substitution and rate heterogeneity was utilized, bootstrap support values of 1000 replicates are shown at the nodes. Names of the ST40 isolates and their origin are indicated at the end of the branches. The highly related ST40 isolates were further zoomed in exemplified by the dotted line and the different scale bar. Metadata are given as follows: Strain no., year of isolation, origin, country: AC, animal colonizer; Al, animal infection; HC, human colonizer; HI, human infection; CU, Cuba; D, Germany; DK, Denmark; ES, Spain; IS, Island; PL, Poland; USA.

by a high bootstrap value (please recall that this separation is only based on the core genome and independent of the presence or absence of MGE). The separation of the two pig commensal isolates D1 and D32 based on core genome data disproves the hypothesis of highly related pig and human endocarditis isolates as derived from PFGE analysis (Figure 1 and [64]).

\section{Analyses of mobile genetic elements}

All isolates featured a modularly structured and differently composed PAI, flanked by phage-related integration and excision genes (data confirmed by long template PCR according to [45]; Additional file 1: Table S1). Of note, genome mapping also revealed that the probiotic Symbioflor 1 strain contains parts of the PAI present in D32 (see Figure 3). However, in this context it is must be noted that the reference isolate D32 itself lacks substantial parts of the PAI (as compared with MMH594) including a number of virulence determinants such as asc-10, the cytolysin operon and the esp gene. Presence of parts of the PAI has been confirmed by long template PCRs performed in advance of genome sequencing experiments. It revealed a number of well-known virulence genes in many ST40 isolates (see above and Additional file 1: Table S1).

Genome analyses of the porcine strain D32 revealed a previously novel and uncharacterised genomic island with a size of 138 kb (location: 1901082 - 2036659, EFD32_1828 - EFD32_1978) integrated at the attachment site of the conjugative vanB transposon in V583 (EF_2282 - EF_2334) $[41,42]$ and the $\mathrm{m}$-inositol (iol) operon in OG1RF, respectively (see Figure 3) [55]. Genome analysis of the probiotic strain Symbioflor indicated that neither the iol operon of the OG1RF nor the vanB transposon of V583 nor the uncharacterised genomic island of the D32 strain was present $[4,65,66]$. Further results of bidirectional BLAST analysis and genome mapping data (Figure 3) revealed that the novel genomic island of D32 was only verifiable in UW7729, an isolate originating from fish. We used SwissProt and BLASTP analyses to identify similarities to capsule-like genes encoded by genes of the novel genomic island in D32, with similarities to genes and gene clusters described for Streptococcus pneumoniae and Bacillus subtilis (Additional file 2: Table S4).

Prophages. We compared phage content by mapping against the D32 reference genome (phiFL4A, phiFL1A, 
B025, phiEF11 [incomplete]). Strain-specific phage patterns were recognised for some of the ST40 isolates, suggesting that prophage content varied independently of the strain background (Figure 3). For example, D32 prophage 1 , showing high similarity to the enterococcal phage phiFL4A (NC_013644), was also present in UW1833, isolated from human urinary tract infection, and in the human colonising strain UW6727. In relation to D32, strains isolated from blood culture and bovine mastitis differed in prophage content, whereas the other ST40 isolates showed a comparably homogeneous level of phage content (in relation to D32).

\section{Plasmid content and classification}

In the context of the 15 de novo sequenced strains, investigation of plasmid content indicated a certain level of diversity independently of the strain background. Plasmids could be classified by the replication initiating gene rep $A$ of the corresponding replicase (rep) families: presence of rep $A$ of the well-described E. faecalis plasmids pAD1, pCF10 and pRE25 was confirmed by Southern hybridisation (not shown). Additionally, sequencing was used to review these results and to differentiate between the amplified conserved repA alleles of the closely related pAD1 and pCF10. The most dominant rep families among the sequenced E. faecalis ST40 were rep2 (pRE25-like) and rep 9 (pCF10-like), found in three (20\%) and six isolates (40\%), respectively.

\section{CRISPR/cas}

Regarding the two CRISPR loci identified in OG1RF, our sequence and PCR results indicated that all of the selected ST40 genomes possessed the CRISPR1-cas and CRISPR2 loci, the latter lacking the functional cas genes. An exception is represented by the genome of UW7729, where only the CRISPR2 locus was present (Additional file 2: Table S5). Our detailed analyses of CRISPR2 loci showed that all strains of the sequenced ST40 subgroup possessed three identical spacers, whereby two of those were also present in OG1RF. In D32 where the CRISPR-cas sequences were completely available, several spacers showed homology with enterococcal phages such as phiEf11, phiFL3A and SAP6, being different from prophages found in the genome sequence of D32 (see above). The exception was phiEf11 which was present in the genome of D32; however, the phage sequence was incomplete (data not shown in details). One spacer in D32 was identical to a hypothetical protein pLG2-0017 of E. faecalis plasmid pLG2 (gb|HQ426665.1|) (rep1 plasmid family; data not shown), which is not in conflict with the plasmid in D32 which is of rep2 plasmid type.

\section{Utilisation of carbon sources}

We postulated that the different origins and habitats of the isolates might be recovered by minor, host-specific differences in their metabolic properties as described for isolates of E. faecium [67]. To determine supposed differences in their metabolic profiles, Biolog MicroArray ${ }^{\text {mix }}$ analyses were performed. For reasons of simplification, data values in Additional file 2: Table S6a and b were replaced by colour codes. Utilisation of various carbon sources under aerobic conditions did not show significant differences between the 15 sequenced E. faecalis ST40 isolates. No obvious association between origin or host and utilisation of different carbon sources was detected.

In general, results were in line with carbon utilisation patterns used for species and genus identification or as described previously $[68,69]$. All strains were capable of growing on trehalose, $\mathrm{N}$-acetyl-glucosamine, glycerol, mannitol, glucose, lactose, sucrose and fructose-6-phosphate. They were all capable of fermenting ribose as a C5 sugar, malate (as an intermediate of the citric acid cycle) and dihydroxyaceton (as an intermediate product of fructose metabolism). No strains grew on melibiose, arabitol and methyl-D-glucoside, as expected. We only noticed a few discrepancies; for instance, according to Devriese and colleagues [69] E. faecalis should not ferment L-arabinose, whereas all ST40 E. faecalis as well as the reference strains V583 and OG1RF did. Utilisation of D-xylose is given as 'mainly negative', but all tested strains including our reference strains were positive. Reference isolate V583 utilised cellobiose, fructose, lactose, glucose, galactose, glycerol, maltose, mannitol, mannose, ribose, sucrose and trehalose as described [42]. One of the minor differences noticed was variable utilisation of myo-inositol (m-inositol). Belonging to one of nine isomers of the inositol group, m-inositol is used as a sole carbon source by many soil and plant microorganisms through degradation into glyceraldehyde-3-phosphate [65]. As already described, the novel and uncharacterised genomic island $(138 \mathrm{~kb})$ of D32 was integrated at the attachment site of the m-inositol operon in OG1RF, which consists of 10 genes. Biolog MicroArray ${ }^{\mathrm{min}}$ analyses showed that OG1RF, but not V583 and D32, was able to utilise minositol. For all the other sequenced ST40 strains, the presence (but not the genomic localisation) of the $i o l$ operon (covering genes encoding enzymes for inositol utilisation) could be confirmed by PCR (data not shown) which was in line with a positive result of $\mathrm{m}$ inositol utilisation in the Biolog MicroArray ${ }^{\mathrm{ma}}$ assay (Additional file 2: Table S6a).

\section{Comparative assessment of the pathogenic potential of closely related $E$. faecalis isolates}

Molecular and phenotypic pre-characterisations revealed a high level of similarity between the porcine, commensal D32 and a human clinical endocarditis isolate UW7709, both from Denmark. Ability to adhere to human epithelial cells and to cause pathogenic effects in 
selected animal models was analysed for these two related ST40 strains.

\section{In vitro growth kinetics}

Over the course of 24 hours, optical density and the corresponding bacterial counts were determined (not shown). Measurement of the optical density suggested that D32 grew faster than UW7709; however, D32 also showed a tendency to clump in liquid culture. Thus, we also determined the bacterial counts, finding a comparatively similar growth rate of E. faecalis strains D32 and UW7709.

\section{In vitro biofilm formation}

E. faecalis strain UW7709 showed a significantly enhanced biofilm production compared with strain D32 (Figure 5). Results of biofilm formation were also compared with the corresponding genotype. Genomes of both strains harboured $f_{s r} B$ and gelE genes in combination with expression of an active metalloprotease GelE. No correlation between the presence of esp or other biofilm-enhancing factors, such as the ebpABC or epa locus, and the in vitro capacity of biofilm formation was detected. The esp gene was absent in both genomes, whereas both genomes harboured the $e b p$ and epa locus.

\section{Adherence to Caco-2 cells}

A monolayer of colonic epithelial cells (Caco-2) was incubated with $E$. faecalis strains D32 and UW7709 to test adhesion to human intestinal cells in vitro. In summary, adherence of D32 to Caco-2 cells was approximately

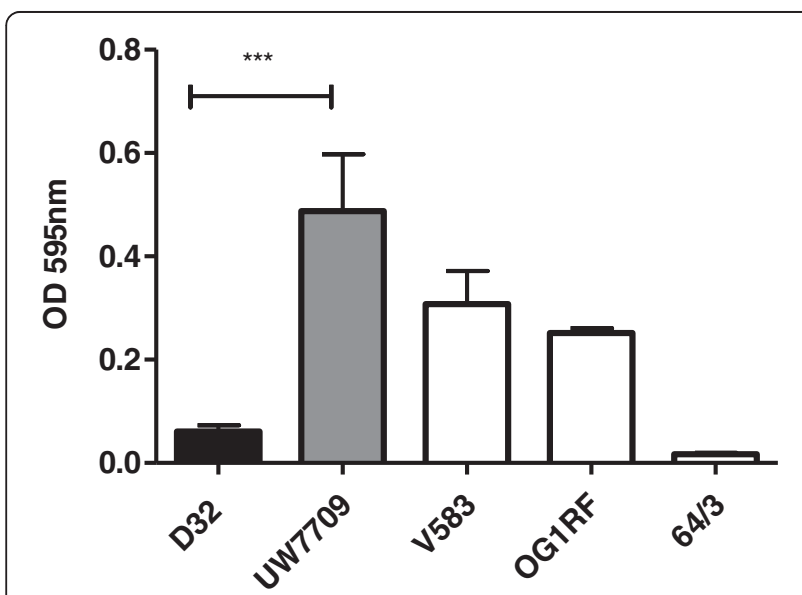

Figure $\mathbf{5}$ In vitro biofilm formation. Biofilm formation of E. faecalis strains D32, UW7709 and the internal controls V583, OG1RF and E. faecium strain 64/3 on a synthetic surface was investigated by using polystyrene plates. After incubation in TSB for 24 hours, produced biofilms of adherent bacteria were stained with crystal violet. Bars represent the mean values of six or three (D32) replicates \pm SEM. *** significant $P$-value $<0.0005$, unpaired two-tailed t-test. three to four times higher than adhesion of UW7709 and similar to V583 (Figure 6).

\section{Galleria mellonella model}

The insect larva G. mellonella is an alternative model for the study of bacteria-host interactions, and shows a complex immune reaction consisting of both cellular and humoral responses. Analysis of pathogenicity of isolates D32 and UW7709 in this model showed that D32 was more rapidly lethal for G. mellonella and pathogenicity of D32 was generally increased in comparison with UW7709 (Figure 7).

\section{Murine bacteraemia model}

With the bacteraemia mouse model, D32 showed significantly enhanced bacterial recovery rates from liver, kidneys, spleen and blood in comparison with UW7709 (Figure 8).

\section{Discussion}

Molecular, genomic and phenotypic strain characterisation reveals a high level of similarity among diverse $E$. faecalis ST40 isolates

SmaI macrorestriction patterns in combination with analyses of the presence and expression of the described virulence-associated genes by PCR revealed a high level of similarity among the rather diverse collection of ST40 isolates (Additional file 1: Table S1). This suggested that strains of this clonal group did not show specific genomic characteristics or differences according to their host, context (commensal/clinical), time, and geography. The finding that major differences in content mainly derive from a diverse acquisition and composition of the various MGE still holds after whole genome sequencing of a subset of 15 isolates from this collection (Figure 4). This indicates that genomic variability within clonal types or lineages may be limited, which is in line with previous reports [49] and which does not contradict the general observation of a high level of recombination at the level of Enterococcus faecalis species. When we performed an SNP-based phylogenetic analysis of the 15 whole genomes an accumulation of SNPs in distinct genomic regions (mainly non-coding regions) pointed to a certain amount of recombination that most probably accompanied the exchange of MGEs. However, the exclusion of recombinational SNPs did not result in a different phylogeny (Figure 4 and Additional file 5: Figure S3), again suggesting that recombination within clonal lineage ST40 is minor.

It has been suggested that 'ecotypes' defined by specific mobile elements may be identified within high-risk lineages or in lineages with variable CRISPR-cas status such as ST40 and ST21 [41]. Environmental isolates exemplified acquisition of distinct plasmids conferring 
A

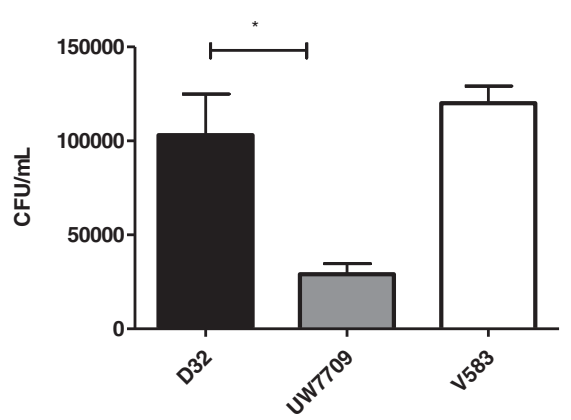

B

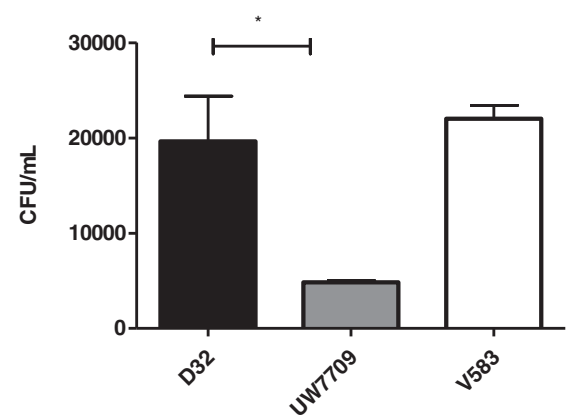

Figure 6 Adhesion ability to Caco-2 cells. To analyze the potential of adhesion, a monolayer of Caco-2 cells was incubated with a bacterial cell to epithelial cell ratio of (A) 1000:1 and (B) 100:1 for two hours with the respective strain. Data represent the mean values \pm SEM. * significant

P-value $<0.05$, unpaired two-tailed t-test.

additional metabolic features such as raffinose fermentation in E. faecium [67]. We did not find any support in a generation of ecotypes or an adaptation to the corresponding origin or host by distinct metabolic features based on genome content which were confirmed by BIOLOG analyses (Additional file 2: Table S6a/b). Nevertheless, utilisation of sugars and other carbon sources was only slightly variable and if so, only variable for individual strains independent of their origin. Differences in carbon utilization patterns could partly be linked to genome content such as for utilising m-inositol (see below). Previous studies concluded that xylose fermentation is limited among $E$. faecalis in general, since, for instance, none of the 10 reference isolates was capable of utilising it [70]. In our case, all sequenced $E$. faecalis isolates were capable of growing on xylose, suggesting a lineage-specific property of ST40 isolates. Recent systemic studies described a model where, in the state of susceptibility to infection by Clostridium difficile caused by high antibiotic selective

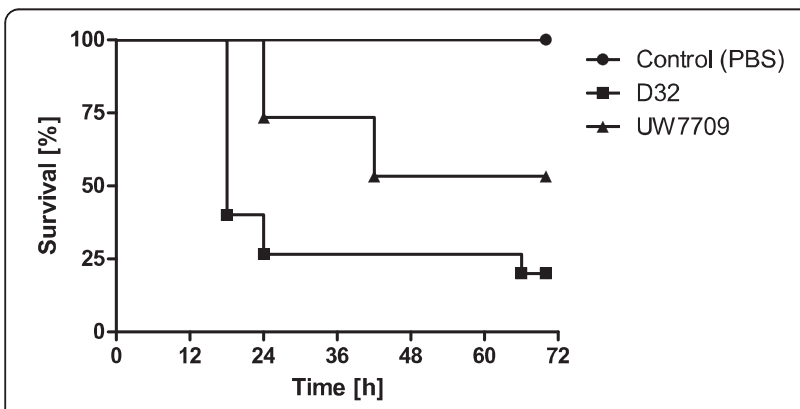

Figure 7 Pathogenicity of E. faecalis D32 and UW7709 in a Galleria mellonella model. Death rates of $G$. mellonella larvae after injection with E. faecalis strains D32 (real infectious dose: $1.7 \times 10^{5}$ CFU per larvae) and UW7709 (real infectious dose: $2.8 \times 10^{5}$ CFU per larvae), respectively. PBS injection served as a negative control. One representative experiment of three independent experiments is shown. Data are displayed by as Kaplan-Meier plot survival curves. Statistical significance $(p<0.05)$ was determined by the Log-rank (Mantel-Cox) test. pressure, sugar alcohols are enriched in the gut metabolome [71]. Bacteria capable of utilising these sugar alcohols may have a definite growth advantage under these specific conditions which is known for E. faecalis and which was dedicated until now to favourable natural and acquired resistances to the used antibiotics. In particular, sugar alcohols such as mannitol and sorbitol increased several hundred-fold under the test conditions and utilisation of these sugars was also strongly positive in all our tested strains (lactitol was only positive among ST40 isolates; Additional file 2: Table $6 \mathrm{a} / \mathrm{b}$ ). However, this does not seem to be a general property, since although all tested $E$. faecalis strains were capable of utilising arabinose they were incapable of utilising arabitol. It should be emphasised that BIOLOG analyses in principle detect utilisation of energy sources via respiration and reduction of the tetrazolium dye and thus do not directly detect growth of microorganisms.

In a previous paper we (G.W., M.Z.) investigated group D streptococci in cases of bovine mastitis in German dairy cows [10]. Strains of ST40 predominated among E. faecalis, emphasising its prevalence as a common and frequent strain type also among dairy cows capable of causing mild and acute clinical cases of bovine mastitis.

Our data showed that previously described virulenceassociated genes, including $f_{s r} A B D C$-regulated gelE, $c y l M$, and esp, as well as clinically relevant antibiotic resistance traits, are also present in ST40 isolates from non-clinical sources. Formation of biofilm (in vitro) could not be correlated with the presence of described and putative biofilm-associated genes such as esp and asc-10. As mentioned by McBride et al. [39] all isolates of this most common sequence type ST40 were nonencapsulated and were also characterised as cps type 1.

Differences between the presence and expression of single markers were especially described for the E. faecalis cytolysin and the gelatinase in previous papers [14,15,72,73]. We did not notice any discrepancy between the presence 
A

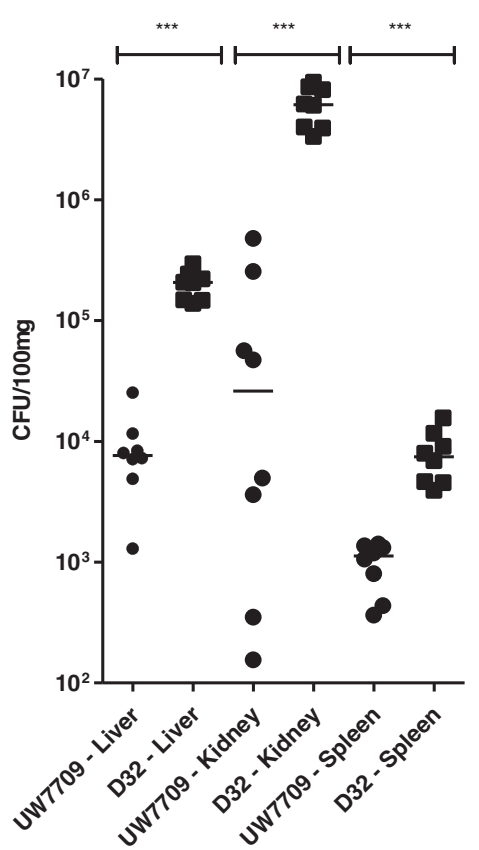

B

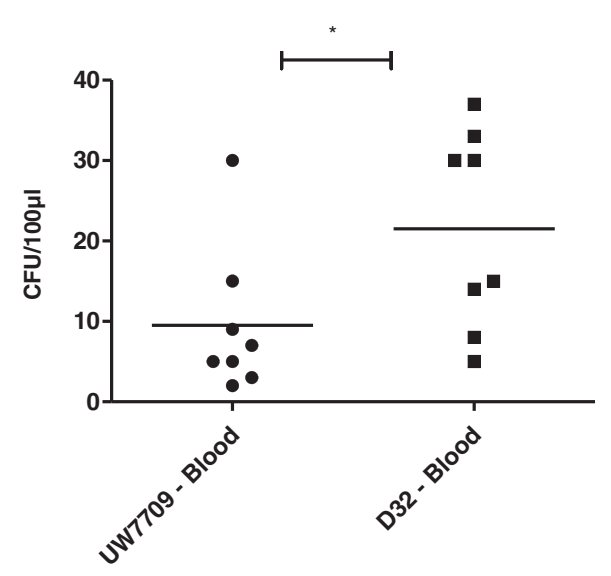

Figure 8 Growth rates of $E$. faecalis UW7709 and D32 in a mouse bacteremia model. Eight female BALB/C mice were infected via the tail vein with E. faecalis strains UW7709 or D32 (5 $\times 10^{8}$ CFU). After 48 hours, mice were sacrificed and bacterial counts in (A) liver, kidneys and spleen, as well as, in (B) blood were determined. Data represent the individual bacterial burdens and the geometric mean values. Asterisks indicate significant $P$ values calculated by using Mann-Whitney test $\left({ }^{*} P<0.05,{ }^{* *} P<0.0005\right)$.

of a gene and its expression in our collection of ST40 E. faecalis strains which was always consistent (Table 4).

\section{Detailed genomic comparisons of 15 ST40 E. faecalis strains identified a modularly structured PAI and a novel genomic island in isolate D32}

On the basis of our previous characterisations, a subset of 15 strains, representing the diversity of the collection, was chosen for de novo pyrosequencing by standard Roche 454 GS-FLX technology. Because no complete ST40 genome sequence was publicly available, we decided to resolve the complete genome sequence of a single pig commensal strain D32 for use as a ST40 template for detailed genome comparisons. After scaffolding with an $8 \mathrm{~kb}$ long-paired end (LPE) library, the remaining gaps and assembly ambiguities ('InDel' errors) were corrected by Sanger sequencing. We gained experience in the use of the two assembly software tools Newbler and Celera, and generated two and one chromosomal scaffolds, respectively. Usage of Celera resulted in fewer misassembled regions, whereas the assembly of repetitive sequences was especially difficult for Newbler. We generated quite positive experience by using PacBio technology for de novo genome sequencing and assemblies and would favour this approach again for upcoming projects. In brief, we were able to generate a single chromosomal scaffold of $2.7 \mathrm{MB}$ by using only the
PacBio approach (long read and short read libraries) of an E. faecium isolate and by using the HGAP algorithm (assemblies done by Celera and polishing by Quiver software; https:/github.com/PacificBiosciences/Bioinformatics-Training/wiki/Large-Genome-Assembly-withPacBio-Long-Reads; project in cooperation with $\mathrm{R}$. Vogelsang, G. Kuhn and F. Boellmann from Pacific Biosciences, previously unpublished data; last access 20.07.2014).

In agreement with McBride et al. [39], we also demonstrated that all of the isolates featured a modularly structured pathogenicity island, varying independently of the strain background and flanked by putative phage-related integrase and excisionase genes [14]. The D32 PAI contained a bile acid hydrolase $(c b h)$ and lactose metabolic pathway genes (lacABCDEFG). It lacked the common markers present in the original prototype PAI of MMH594 which were mainly associated with virulence, like esp, the asc-10 gene and the cytolysin operon. In the case of D32, composition of the PAI may suggest a putative adaptation to pig's intestine by the presence of bile acid hydrolase $(c b h)$ and the complete lactose metabolic pathway genes (lacABCDEFG). Detailed analyses of the genetic components of the PAIs of the 14 E. faecalis ST40 draft genomes were limited by the existence of intra-chromosomal gaps. However, the presence and absence of PAI components/clusters were available from results of long PCR analyses, which showed variability 
independent from the host (Additional file 1: Table S1). Variability is derived from partial or complete mobility of the PAI, as demonstrated recently [45,46,74]. These results again emphasise that the PAI evolves through HGT and recombination in a much faster way than the relatively conserved core genome [32,41].

A so far unknown genomic island was identified as integrated within the genome of the porcine commensal strain D32. Apart from several genes with unknown identity, it contains a genomic cluster probably associated with exopolysaccharide synthesis. Consisting of different sugars, the extracellular polysaccharides (EPS) are polymeric complex structures covalently bound to the cell surface or released in its environment $[75,76]$. Importantly, conserved E. faecalis cell wall polysaccharides are the rhamnopolysaccharide Epa, encoded by the enterococcal polysaccharide biosynthesis locus epa, and the capsular polysaccharide Cps (cps cluster), consisting of galactose, glucose and phosphate [77]. All ST40 strains were non-encapsulated and are grouped as CPS type 1 . Although the presence of cps genes does not necessarily result in the expression of the corresponding capsule phenotype [78], genes related to cell surface structures are enriched with strains of CC2 [35,39,79].

Investigations regarding the integration site of the novel GI showed that it is integrated within a conserved attL/attR attachment site, previously described as a 'hot spot' for rearrangements or new integrations. It is suggested that two different integration events resulted in the presence of an iol operon in OG1RF and a conjugative $\operatorname{vanB}$ transposon in V583 $[42,65]$. The attachment region is missing in the Symbioflor 1 strain [4]. Our sequencing results demonstrated that all of the ST40 strains, with the exception of D32, harbour an iol operon comparable to OG1RF, coupled with identical phenotypes of m-inositol utilisation (see Additional file 2: Table S6a/b). M-inositol is a substance abundant in nature and its utilisation was suggested recently as an auxiliary trait promoting fitness in lactic acid bacteria such as lactobacilli [80]. Genome data in combination with results of Biolog MicroArray ${ }^{\text {Tim }}$ analyses of the strain UW7729, isolated from fish, suggested that the iol operon was co-integrated next to the novel GI. However, the presence of intra-chromosomal gaps within this genomic region limited our opportunities to display the colocalisation in more molecular detail.

Resolving the plasmid and phage content and prevalence of genomic islands requires techniques in addition to illumina sequencing

Suitable software tools which filter for plasmid sequences, generated by 454 or the Illumina's Solexa sequencing technology, are limited, as are approaches to identifying entire plasmids from de novo assembled genomic DNA. Thus, 'classical' molecular methods, such as plasmid isolation and analysis of the plasmid size by S1 nuclease PFGE in combination with PCR- and hybridisation-based rep-typing, were used as more meaningful and informative approaches. In combination with genomic mapping of the ST40 draft genomes against the D32 template, results revealed a comparably high level of diversity in plasmid content [81] and strain-specific phage patterns (Figure 3). Integration of bacteriophages might have a significant impact on niche adaptation. Duerkop et al. [82] hypothesised that bacteriophages, especially a composition of different bacteriophages, cause bacterial dominance within the microbial ecosystem, where competition for nutrients especially plays a crucial role. Integration of the bacteriophage into a promoter region might result in an increase of expression of putative virulence-associated genes [83]. Surface-exposed wall teichoic acids might be used as specific identification markers for bacteriophages, enabling HGT of virulence and resistance genes across genusspecific barriers of Gram-positive pathogens [84].

The rep-typing scheme for determining plasmid types demonstrated that rep-types 2 and 9 were predominant in the ST40 strain collection but disclosed also the presence of some new plasmids $[55,85,86]$. These results indicated that recombination and rearrangements of chromosomal and other plasmid DNA might contribute to mosaic-related structures, whereby an adaptation to changing environmental conditions is mediated [87].

A subset of ST40 strains has been analysed at a partner's laboratory by microarray hybridisation analyses of isolates of E. faecalis and E. faecium dedicated to detecting specifically mobile genetic elements and resistance genes for another study [88]. Only isolates of ST40 harboured possible functional CRISPR-cas systems which were not detected, for instance, in hospital-associated $E$. faecalis ST6 isolates (CC2). Whereas resistance determinants were evenly distributed among different strains and ST types, isolates of ST40 in particular showed an accumulation of antibiotic, metal and biocide resistance determinants. A link to the presence of corresponding mobile elements such as transposons, conjugative transposons and plasmids frequently carrying these resistance genes was noticeable, suggesting that the presence of CRISPR-cas is highly specific and does not generally exclude accumulation of resistance determinants or mobile genetic elements as suggested recently [89].

\section{Using different in vivo models to analyse adhesion and pathogenicity revealed a higher pathogenic potential of D32 in comparison with UW7709}

Increased adherence to biotic surfaces such as that of D32 to Caco-2 cells (Figure 6) does not necessarily correlate with an enhanced capacity of biofilm formation in vitro (regarding abiotic surfaces; Additional file 3: Figure S1), a 
fact that has been addressed already in previous papers [90,91]. So far described regulators, such as Fsr [92], EbpR [93], EbrA [94], and PerA [95], are suggested to influence biofilm formation and, as demonstrated recently, other genes of the conserved core genome could also be involved in biofilm expression and gene regulation [94].

A number of different in vivo models were utilised to compare the in vivo survival and pathogenic potential of the two related E. faecalis strains D32 and UW7709. Growth in vitro did not differ between these two strains. In the insect larvae Galleria model, D32 was more rapidly lethal and pathogenicity of D32 was generally higher compared with UW7709 (Figure 7). Similar results were generated when recovery rates of bacterial counts were compared for the murine bacteraemia model with significantly enhanced amounts of D32 in comparison with UW7709 isolated from liver, kidneys, spleen and blood (Figure 8). Results were confirmed with a rat endocarditis model in a partner's lab (T.S and J. H.) (not shown) [96]. During the course of this study, we tested two other avian models of infection at a partner's laboratory (H.M.H, R.H.), which also gave similar results (not shown) [97]. Taken together, all used animal models of infection and pathogenicity proclaimed a higher pathogenic potential of the pig isolate D32 in comparison with the human endocarditis isolate UW7709. Obvious differences in the genome content of both isolates mainly included the presence or absence of a few prophage sequences and the novel genomic island in D32 potentially encoding a cluster of polysaccharide capsule genes (Figure 3). Arguing that this novel genomic island increases pathogenicity of D32 in the tested animal models will require targeted knockout experiments by deleting this novel $134 \mathrm{~kb}$ island in the strain background of D32 and subsequent analysis of the behaviour of isogenic strain pairs in the different models; such experiments are planned.

Preliminary results of secretome analyses suggested differences in the extracellular proteome of the two related strains D32 and UW7709 [98]. Differences in the secretome and the above-mentioned putative novel exopolysaccharide cluster could explain why D32 in comparison with UW7709 showed significantly different behaviour in the presented bacteria-host models $[99,100]$.

\section{Conclusion}

Our detailed molecular and phenotypic analyses of $42 E$. faecalis strains of MLST type ST40 and the genomic analyses of a subset of 15 isolates revealed a minor level of genomic diversity. Isolates are highly related regarding the core genome and only demonstrated variable accessory genome content in respect of the presence and composition of the E. faecalis PAI, plasmids and phages, independently of their corresponding animal or human background or the context (colonisation/infection). We were unable to find any indication of niche adaptation associated with the supposed origin or clinical context of distinct ST40 strains. The animal isolate D32, whose genome was completely resolved during this study, contained a novel genomic island of $138 \mathrm{~kb}$, putatively involved in exopolysaccharide synthesis (capsule formation, perhaps?). D32 showed enhanced pathogenic potential in various animal models compared with the related human endocarditis isolate UW7709. Our hypothesis suggests that different behaviour of D32 and UW7709 is associated with the presence of this novel genomic island, a supposition which needs to be proven in future analyses.

\section{Animal experiments}

The animal welfare committees of the university of Freiburg (Regierungspräsidium Freiburg Az 35/9185.81/ G-07/15) approved all animal experiments (sepsis model G-07/15; rat endocarditis model G-07/72; urinary tract infection model G-11/118).

\section{Availability of supporting data}

All supporting information is deposited as Supplementary Figures or Tables.

\section{Endnote}

${ }^{a}$ http://www.ecdc.europa.eu/en/publications/surveillance_reports/annual_epidemiological_report/Pages/epi_index.aspx

\section{Additional files}

Additional file 1: Table S1. Main characteristics of the analysed 42 ST40 E. faecalis isolates.

Additional file 2: Table S2. Primers used for the amplification of antibiotic resistance genes, virulence genes, parts of the $E$. faecalis PAI, plasmid replicase genes and other genes. Table S3. Quality report of 454 sequencing data assembled with Newbler software. Table S4. SwissProt and BLASTP analyses of a putative capsule-encoding region within the $E$. faecalis D32 genomic island. Table S5. Identification of CRISPR loci in selected E. faecalis ST40 strains by PCR. Table S6. Aerobic utilization of carbon sources of Biolog MicroArray ${ }^{\mathrm{TM}}$ PM01 and PM02.

Additional file 3: Figure S1. Capability of E. faecalis isolates of ST40 to form biofilm in vitro on polystyrene microtiter plates (Greiner Bio-one, Germany and Corning Inc., NY, USA). Isolates from UTI are marked with dark blue as well as isolates from endocarditis (blue). Strains E. faecalis V583, OG1RF and OG1RFK12 (OG1RF including the PAl and plasmid pLG2 [45] were used as reference isolates as well as E. faecium 64/3 [54] as a negative control (all marked with black). Isolate UW7742 (=D32) is the completely sequenced ST40 reference isolate showing a lower biofilm forming capability.

Additional file 4: Figure S2. S1 nuclease analysis resolving plasmid content of 18 ST40 E. faecalis isolates. This representative gel of S1 nuclease PFGE showed the presence of none, one or two linearized plasmids. A red arrow points at a single plasmid band of the sequenced E. faecalis strain D32 (size of circa $75 \mathrm{~kb}$ ). The upper bands (migrating above the $674 \mathrm{~kb}$ band of NCTC8325), visible in all lanes, correspond to undigested chromosomal DNA. NCTC8325 applies to Smal-digested 
genomic DNA of S. aureus NCTC8325 used as a size marker in PFGE analysis.

Additional file 5: Figure S3. Phylogenetic analysis of 15 E. faecalis ST40 genomes resulting from mapping illumina reads against the D32 reference genome. Solexa single reads were mapped onto the reference sequence of $E$. faecalis D32 by utilizing a mapping pipeline based on bwa (Steglich et al., previously unpublished [see Materials]). Altogether $10,4 \%$ of ambiguous sites were detected compared to the reference genome and excluded from further analysis as well as SNPs resulting from recombination, thereby producing 1481 SNPs for phylogenetic calculations by the PhyML algorithm (seaview program) with a bootstrap of 1000. Metadata are given as follows: Strain no., year of isolation, origin, country: AC, animal colonizer; Al, animal infection; $\mathrm{HC}$, human colonizer; HI, human infection; CU, Cuba; D, Germany; DK, Denmark; ES, Spain; IS, Island; PL, Poland; USA.

\section{Competing interests}

The authors declare that they have no competing interests.

\section{Authors' contributions}

Designed and discussed the study: GW, WW, MZ, PT, DJ, TH; performed the strain pre-assessment and sample preparation: MSo, ES, JL, LJ, PRG, DQ, TM, GW; performed most of the genotypic and phenotypic laboratory experiments: MZ; prepared samples for NGS and performed the NGS sequencing and primary data analyses: MZ, SV, RD, AN; run NGS data assembly, annotation and phylogenetic analyses: $C K, T H, T C, J B I, W D, A N, B L, A G, S V, R D, J B e$, MSt, UN; contributed to and/or performed the animal experiments: MZ, DW $\mathrm{TS}, \mathrm{JH}, \mathrm{RH}, \mathrm{HMH}$; contributed to and/or performed the BIOLOG analysis: MZ, $\mathrm{KSH}$, DS; wrote the manuscript: MZ, GW. All authors read and approved the final manuscript.

\section{Acknowledgements}

We would like to thank our cooperation partners for their support and fruitful discussions. A special thanks to Eurofins MWG-Operon and GATC, Konstanz for 454 sequencing services rendered. Special thanks to Dr. Henning Böhme (Wernigerode, D), Prof. Dr. Nobumichi Kobayashi (Sapporo, JP), and all of the other clinical laboratories in Germany for the kind gift of strains. The study was supported by a grant from the Robert Koch Institute and the Federal Ministry of Education and Research (BMBF; No. 0315833C) to M.Z. Excellent technical assistance is acknowledged for Carola Fleige, Uta Geringer and Christine Günther.

\section{Author details}

'Division of Nosocomial Pathogens and Antibiotic Resistances, Department of Infectious Diseases, Robert Koch Institute, Wernigerode Branch, Burgstr. 37, D-38855 Wernigerode, Germany. ${ }^{2}$ Present address: Institute for Pathology, Hannover Medical School (MHH), Hannover, Germany. ${ }^{3}$ Functional Genomics of Bacterial Pathogens, Institute for Medical Microbiology, Justus Liebig University Giessen and German Center for Infection Research (DZIF), Partner site Giessen-Marburg-Langen, Campus Giessen, Giessen, Germany. ${ }^{4}$ Max Planck Institute for Heart and Lung Research, Bad Nauheim, Germany. ${ }^{5}$ Center for Biotechnology (CeBiTec)/University of Bielefeld, Bielefeld, Germany. Institute for Bioinformatics and Systems Biology, Justus Liebig University Giessen, Giessen, Germany. ${ }^{7}$ Division of Infectious Diseases, Department of Medicine, University Hospital Freiburg, Freiburg, Germany. ${ }^{8}$ Institute for Biochemistry, Biotechnology and Bioinformatics, Technische Universität Braunschweig, Braunschweig, Germany. 'Robert Koch Institute, ZBS 1 Highly Pathogenic Viruses, Centre for Biological Threats and Special Pathogens, Berlin, Germany. ${ }^{10}$ Division of Pediatric Infectious Diseases, Hauner Children's Hospital, Ludwig-Maximilians University Munich, Munich, Germany. ${ }^{11}$ Goettingen Genomics Laboratory, Georg August University, Goettingen, Germany. ${ }^{12}$ Department of Veterinary Medicine, Institute for Poultry Diseases, Free University Berlin, Berlin, Germany. ${ }^{13}$ Institute for Microbiology, Technische Universität Braunschweig, Braunschweig, Germany. ${ }^{14}$ Laboratory of Microbial Gene Technology and Food Microbiology, The Norwegian University of Life Sciences, Ås, Norway. ${ }^{15}$ National Medicines Institute, Warsaw, Poland. ${ }^{16}$ Statens Serum Institute, Copenhagen, Denmark. ${ }^{17}$ Division of Microbiology, National Food Institute, Danish Technical University, Copenhagen, Denmark. ${ }^{18}$ Department of Microbiology, Hospital Ramon y Cajal, Madrid, Spain. ${ }^{19}$ Instituto de Medicina Tropical Pedro Kourí,
Servicio de Bacteriología-Micología, La Habana, Cuba. ${ }^{20}$ Department of Medical Biology, Faculty of Health Sciences, Research Group for Host Microbe Interactions, University of Tromsø, Tromsø, Norway. ${ }^{21}$ Leibniz-Institut DSMZ - Deutsche Sammlung von Mikrorganismen und Zellkulturen GmbH, Braunschweig, Germany.

Received: 23 July 2014 Accepted: 20 February 2015 Published online: 12 March 2015

\section{References}

1. Kondoh M, Fukada K, Fujikura D, Shimada T, Suzuki Y, Iwai A, et al. Effect of water-soluble fraction from lysozyme-treated Enterococcus faecalis FK-23 on mortality caused by influenza A virus in mice. Viral Immunol. 2012;25(1):86-90.

2. Wang Z, Burwinkel M, Chai W, Lange E, Blohm U, Breithaupt A, et al. Dietary Enterococcus faecium NCIMB 10415 and zinc oxide stimulate immune reactions to trivalent influenza vaccination in pigs but do not affect virological response upon challenge infection. PLoS One. 2014;9(1):e87007.

3. Szabo I, Wieler LH, Tedin K, Scharek-Tedin L, Taras D, Hensel A, et al. Influence of a probiotic strain of Enterococcus faecium on Salmonella enterica serovar Typhimurium DT104 infection in a porcine animal infection model. Appl Environ Microbiol. 2009;75(9):2621-8.

4. Fritzenwanker M, Kuenne C, Billion A, Hain T, Zimmermann K, Goesmann A, et al. Complete genome sequence of the probiotic Enterococcus faecalis Symbioflor 1 clone DSM 16431. Genome Announcements. 2013;1(1).

5. Khan H, Flint S, Yu PL. Enterocins in food preservation. Int J Food Microbiol. 2010;141(1-2):1-10.

6. Nallapareddy SR, Singh KV, Sillanpaa J, Garsin DA, Hook M, Erlandsen SL, et al. Endocarditis and biofilm-associated pili of Enterococcus faecalis. J Clin Invest. 2006;116(10):2799-807.

7. Singh KV, Nallapareddy SR, Murray BE. Importance of the ebp (endocarditisand biofilm-associated pilus) locus in the pathogenesis of Enterococcus faecalis ascending urinary tract infection. J Infect Dis. 2007;195(11):1671-7.

8. Singh KV, Nallapareddy SR, Sillanpaa J, Murray BE. Importance of the collagen adhesin ace in pathogenesis and protection against Enterococcus faecalis experimental endocarditis. PLoS Pathog. 2010;6(1):e1000716.

9. Thurlow LR, Thomas VC, Narayanan S, Olson S, Fleming SD, Hancock LE. Gelatinase contributes to the pathogenesis of endocarditis caused by Enterococcus faecalis. Infect Immun. 2010;78(11):4936-43.

10. Werner G, Fleige C, Fessler AT, Timke M, Kostrzewa M, Zischka M, et al. Improved identification including MALDI-TOF mass spectrometry analysis of group D streptococci from bovine mastitis and subsequent molecular characterization of corresponding Enterococcus faecalis and Enterococcus faecium isolates. Vet Microbiol. 2012;160(1-2):162-9.

11. Van Tyne D, Martin MJ, Gilmore MS. Structure, function, and biology of the Enterococcus faecalis cytolysin. Toxins. 2013;5(5):895-911.

12. Coburn PS, Pillar CM, Jett BD, Haas W, Gilmore MS. Enterococcus faecalis senses target cells and in response expresses cytolysin. Science. 2004;306 (5705):2270-2.

13. Clewell DB. Properties of Enterococcus faecalis plasmid pAD1, a member of a widely disseminated family of pheromone-responding, conjugative, virulence elements encoding cytolysin. Plasmid. 2007;58(3):205-27.

14. Shankar N, Baghdayan AS, Gilmore MS. Modulation of virulence within a pathogenicity island in vancomycin-resistant Enterococcus faecalis. Nature. 2002;417(6890):746-50.

15. Hallgren A, Claesson C, Saeedi B, Monstein HJ, Hanberger H, Nilsson LE. Molecular detection of aggregation substance, enterococcal surface protein, and cytolysin genes and in vitro adhesion to urinary catheters of Enterococcus faecalis and E. faecium of clinical origin. Int J Med Microbiol. 2009;299(5):323-32.

16. Jett BD, Jensen HG, Nordquist RE, Gilmore MS. Contribution of the pAD1encoded cytolysin to the severity of experimental Enterococcus faecalis endophthalmitis. Infect Immun. 1992;60(6):2445-52.

17. Qin X, Singh KV, Weinstock GM, Murray BE. Effects of Enterococcus faecalis fsr genes on production of gelatinase and a serine protease and virulence. Infect Immun. 2000;68(5):2579-86.

18. Bourgogne A, Hilsenbeck SG, Dunny GM, Murray BE. Comparison of OG1RF and an Isogenic fsrB Deletion Mutant by Transcriptional Analysis: the Fsr System of Enterococcus faecalis is More than the Activator of Gelatinase and Serine Protease. J Bacteriol. 2006;188(8):2875-84. 
19. Park SY, Shin YP, Kim CH, Park HJ, Seong YS, Kim BS, et al. Immune evasion of Enterococcus faecalis by an extracellular gelatinase that cleaves C3 and iC3b. J Immunol. 2008;181(9):6328-36.

20. Galloway-Pena JR, Bourgogne A, Qin X, Murray BE. Diversity of the fsr-gelE region of the Enterococcus faecalis genome but conservation in strains with partial deletions of the fsr operon. Appl Environ Microbiol. 2011;77(2):442-51.

21. Qin $X$, Singh KV, Weinstock GM, Murray BE. Characterization of $f s r$, a regulator controlling expression of gelatinase and serine protease in Enterococcus faecalis OG1RF. J Bacteriol. 2001;183(11):3372-82.

22. Sava IG, Zhang F, Toma I, Theilacker C, Li B, Baumert TF, et al. Novel interactions of glycosaminoglycans and bacterial glycolipids mediate binding of enterococci to human cells. J Biol Chem. 2009;284(27):18194-201.

23. Theilacker C, Sanchez-Carballo P, Toma I, Fabretti F, Sava I, Kropec A, et al. Glycolipids are involved in biofilm accumulation and prolonged bacteraemia in Enterococcus faecalis. Mol Microbiol. 2009;71(4):1055-69.

24. Thurlow LR, Thomas VC, Hancock LE. Capsular polysaccharide production in Enterococcus faecalis and contribution of CpsF to capsule serospecificity. J Bacteriol. 2009;191(20):6203-10

25. Hendrickx AP, van Luit-Asbroek M, Schapendonk CM, van Wamel WJ, Braat JC, Wijnands LM, et al. SgrA, a nidogen-binding LPXTG surface adhesin implicated in biofilm formation, and EcbA, a collagen binding MSCRAMM, are two novel adhesins of hospital-acquired Enterococcus faecium. Infect Immun. 2009:77(11):5097-106.

26. Hendrickx AP, Willems RJ, Bonten MJ, van SW. LPxTG surface proteins of enterococci. Trends Microbiol. 2009;17(9):423-30.

27. Shankar N, Lockatell CV, Baghdayan AS, Drachenberg C, Gilmore MS, Johnson DE. Role of Enterococcus faecalis surface protein Esp in the pathogenesis of ascending urinary tract infection. Infect Immun. 2001;69(7):4366-72.

28. Tendolkar PM, Baghdayan AS, Gilmore MS, Shankar N. Enterococcal surface protein, Esp, enhances biofilm formation by Enterococcus faecalis. Infect Immun. 2004:72(10):6032-9.

29. Tendolkar PM, Baghdayan AS, Shankar N. The N-terminal domain of enterococcal surface protein, Esp, is sufficient for Esp-mediated biofilm enhancement in Enterococcus faecalis. J Bacteriol. 2005;187(17):6213-22.

30. van Schaik W, Top J, Riley DR, Boekhorst J, Vrijenhoek JEPV, Schapendonk $\mathrm{CME}$, et al. Pyrosequencing-based comparative genome analysis of the nosocomial pathogen Enterococcus faecium and identification of a large transferable pathogenicity island. BMC Genomics. 2010;11:239.

31. Shankar N, Baghdayan AS, Willems R, Hammerum AM, Jensen LB. Presence of pathogenicity island genes in Enterococcus faecalis isolates from pigs in Denmark. J Clin Microbiol. 2006;44(11):4200-3.

32. McBride SM, Coburn PS, Baghdayan AS, Willems RJL, Grande MJ, Shankar N, et al. Genetic variation and evolution of the pathogenicity island of Enterococcus faecalis. J Bacteriol. 2009;191(10):3392-402.

33. Olsen $\mathrm{RH}$, Schonheyder $\mathrm{HC}$, Christensen H, Bisgaard M. Enterococcus faecalis of human and poultry origin share virulence genes supporting the zoonotic potential of E. faecalis. Zoonoses Public Health. 2012;59(4):256-63.

34. Vebø HC, Solheim M, Snipen L, Nes IF, Brede DA. Comparative genomic analysis of pathogenic and probiotic Enterococcus faecalis isolates, and their transcriptional responses to growth in human urine. PLoS One. 2010;5(8):e12489

35. Solheim M, Aakra A, Snipen LG, Brede DA, Nes IF. Comparative genomics of Enterococcus faecalis from healthy Norwegian infants. BMC Genomics. 2009;10:194.

36. Dunny GM. The peptide pheromone-inducible conjugation system of Enterococcus faecalis plasmid pCF10: cell-cell signalling, gene transfer, complexity and evolution. Phil Transactions Royal Soc London Series B, Biol Sci. 2007;362(1483):1185-93.

37. Sussmuth SD, Muscholl-Silberhorn A, Wirth R, Susa M, Marre R, Rozdzinski E. Aggregation substance promotes adherence, phagocytosis, and intracellular survival of Enterococcus faecalis within human macrophages and suppresses respiratory burst. Infect Immun. 2000;68(9):4900-6.

38. Rozdzinski E, Marre R, Susa M, Wirth R, Muscholl-Silberhorn A. Aggregation substance-mediated adherence of Enterococcus faecalis to immobilized extracellular matrix proteins. Microb Pathog. 2001;30(4):211-20.

39. McBride SM, Fischetti VA, Leblanc DJ, Moellering Jr RC, Gilmore MS. Genetic diversity among Enterococcus faecalis. PLoS One. 2007;2(7):e582.

40. Clewell DB, Weaver KE, Dunny GM, Coque TM, Francia MV, Hayes F. Extrachromosomal and mobile elements in enterococci: transmission, maintenance, and epidemiology. In: Gilmore MS, Clewell DB, Ike Y, Shankar
$\mathrm{N}$, editors. Enterococci: from commensals to leading causes of drug resistant infection. Boston: Massachusetts Eye and Ear Infirmary; 2014.

41. Palmer KL, Godfrey P, Griggs A, Kos VN, Zucker J, Desjardins C, et al. Comparative genomics of enterococci: variation in Enterococcus faecalis, clade structure in E. faecium, and defining characteristics of E. gallinarum and E. casseliflavus. mBio. 2012;3(1):e00318-00311.

42. Paulsen IT, Banerjei L, Myers GS, Nelson KE, Seshadri R, Read TD, et al. Role of mobile DNA in the evolution of vancomycin-resistant Enterococcus faecalis. Science. 2003;299(5615):2071-4.

43. Zhu W, Clark NC, McDougal LK, Hageman J, McDonald LC, Patel JB. Vancomycin-resistant Staphylococcus aureus isolates associated with Inc18-like vanA plasmids in Michigan. Antimicrob Agents Chemother. 2008:52(2):452-7.

44. Zhu W, Murray PR, Huskins WC, Jernigan JA, MCDonald LC, Clark NC, et al. Dissemination of an Enterococcus Inc18-like vanA plasmid, associated with vancomycin-resistant Staphylococcus aureus. Antimicrob Agents Chemother. 2010;54(10):4314

45. Laverde Gomez JA, Hendrickx AP, Willems RJ, Top J, Sava I, Huebner J, et al. Intra- and interspecies genomic transfer of the Enterococcus faecalis pathogenicity island. PLoS One. 2011;6(4):e16720.

46. Manson JM, Hancock LE, Gilmore MS. Mechanism of chromosomal transfer of Enterococcus faecalis pathogenicity island, capsule, antimicrobial resistance, and other traits. Proc Natl Acad Sci USA. 2010;107:12269-74.

47. de Been M, van Schaik W, Cheng L, Corander J, Willems RJ. Recent recombination events in the core genome are associated with adaptive evolution in Enterococcus faecium. Genome Biol Evol. 2013;5(8):1524-35

48. Lebreton F, van Schaik W, Manson McGuire A, Godfrey P, Griggs A, Mazumdar $\checkmark$, et al. Emergence of epidemic multidrug-resistant Enterococcus faecium from animal and commensal strains. mBio. 2013:4(4):pii: e00534-00513.

49. Willems RJ, Top J, van Schaik W, Leavis H, Bonten M, Siren J, et al. Restricted gene flow among hospital subpopulations of Enterococcus faecium. mBio. 2012;3(4):e00151-00112.

50. Poulsen LL, Bisgaard M, Son NT, Trung NV, An HM, Dalsgaard A. Enterococcus faecalis clones in poultry and in humans with urinary tract infections, Vietnam. Emerg Infect Dis. 2012;18(7):1096-100.

51. Kuch A, Willems RJ, Werner G, Coque TM, Hammerum AM, Sundsfjord A, et al. Insight into antimicrobial susceptibility and population structure of contemporary human Enterococcus faecalis isolates from Europe. J Antimicrob Chemother. 2012;67(3):551-8.

52. Werner G, Freitas AR, Coque TM, Sollid JE, Lester C, Hammerum AM, et al. Host range of enterococcal vanA plasmids among Gram-positive intestinal bacteria. J Antimicrob Chemother. 2010;66(2):273-82.

53. Ruiz-Garbajosa P, Bonten MJ, Robinson DA, Top J, Nallapareddy SR, Torres C, et al. Multilocus sequence typing scheme for Enterococcus faecalis reveals hospital-adapted genetic complexes in a background of high rates of recombination. J Clin Microbiol. 2006:44(6):2220-8.

54. Laverde Gomez JA, van SW, Freitas AR, Coque TM, Weaver KE, Francia MV, et al. A multiresistance megaplasmid pLG1 bearing a hyl(Efm) genomic island in hospital Enterococcus faecium isolates. Int J Med Microbiol. 2010:301:165-75

55. Zischka M, Kuenne C, Blom J, Dabrowski PW, Linke B, Hain T, et al. Complete genome sequence of the porcine isolate Enterococcus faecalis D32. J Bacteriol. 2012;194(19):5490-1.

56. Alikhan NF, Petty NK, Ben Zakour NL, Beatson SA. BLAST Ring Image Generator (BRIG): simple prokaryote genome comparisons. BMC Genomics. 2011;12:402.

57. Angiuoli SV, Salzberg SL. Mugsy: fast multiple alignment of closely related whole genomes. Bioinformatics. 2011;27(3):334-42.

58. Sahl JW, Matalka MN, Rasko DA. Phylomark, a tool to identify conserved phylogenetic markers from whole-genome alignments. Appl Environ Microbiol. 2012;78(14):4884-92.

59. Stamatakis A. RAxML-VI-HPC: maximum likelihood-based phylogenetic analyses with thousands of taxa and mixed models. Bioinformatics. 2006;22(21):2688-90.

60. Gripp E, Hlahla D, Didelot X, Kops F, Maurischat S, Tedin K, et al. Closely related Campylobacter jejuni strains from different sources reveal a generalist rather than a specialist lifestyle. BMC Genomics. 2011;12:584.

61. Park SY, Kim KM, Lee JH, Seo SJ, Lee IH. Extracellular gelatinase of Enterococcus faecalis destroys a defense system in insect hemolymph and human serum. Infect Immun. 2007;75(4):1861-9. 
62. Mukherjee K, Altincicek B, Hain T, Domann E, Vilcinskas A, Chakraborty T. Galleria mellonella as a model system for studying Listeria pathogenesis. Appl Environ Microbiol. 2010;76(1):310-7.

63. Hufnagel M, Koch S, Creti R, Baldassarri L, Huebner J. A putative sugar-binding transcriptional regulator in a novel gene locus in Enterococcus faecalis contributes to production of biofilm and prolonged bacteremia in mice. J Infect Dis. 2004;189(3):420-30.

64. Larsen J, Schonheyder HC, Singh KV, Lester CH, Olsen SS, Porsbo LJ, et al. Porcine and human community reservoirs of Enterococcus faecalis, Denmark. Emerg Infect Dis. 2011;17(12):2395-7.

65. Bourgogne A, Garsin DA, Qin X, Singh KV, Sillanpaa J, Yerrapragada S, et al. Large scale variation in Enterococcus faecalis illustrated by the genome analysis of strain OG1RF. Genome Biol. 2008;9(7):R110.

66. Domann E, Hain T, Ghai R, Billion A, Kuenne C, Zimmermann K, et al. Comparative genomic analysis for the presence of potential enterococcal virulence factors in the probiotic Enterococcus faecalis strain Symbioflor 1. Int J Med Microbiol. 2007;297(7-8):533-9.

67. Zhang X, Vrijenhoek JEP, Bonten MJM, Willems RJ, van Schaik W. A large conjugative plasmid confers the ability to utilize a-galactoside sugars as carbon source in E. faecium. ESCMID Conference on Enterococci: from Animals to Man, Conference Proceedings 2009, -(-):34-34.

68. Ramsey M, Hartke A, Huycke M. The physiology and metabolism of enterococci. In: Gilmore MS, Clewell DB, Ike Y, Shankar N, editors. Enterococci: from commensals to leading causes of drug resistant infection. Boston: Massachusetts Eye and Ear Infirmary; 2014.

69. Devriese LA, Pot B, Collins MD. Phenotypic identification of the genus Enterococcus and differentiation of phylogenetically distinct enterococcal species and species groups. J Appl Bacteriol. 1993;75(5):399-408.

70. Aakra A, Nyquist OL, Snipen L, Reiersen TS, Nes IF. Survey of genomic diversity among Enterococcus faecalis strains by microarray-based comparative genomic hybridization. Appl Environ Microbiol. 2007;73(7):2207-17.

71. Theriot CM, Koenigsknecht MJ, Carlson Jr PE, Hatton GE, Nelson AM, Li B, et al. Antibiotic-induced shifts in the mouse gut microbiome and metabolome increase susceptibility to Clostridium difficile infection. Nature Comm. 2014,5:3114.

72. Rosa R, Creti R, Venditti M, D'Amelio R, Arciola CR, Montanaro L, et al. Relationship between biofilm formation, the enterococcal surface protein (Esp) and gelatinase in clinical isolates of Enterococcus faecalis and Enterococcus faecium. FEMS Microbiol Lett. 2006;256(1):145-50.

73. Teixeira N, Santos S, Marujo P, Yokohata R, lyer VS, Nakayama J, et al. The incongruent gelatinase genotype and phenotype in Enterococcus faecalis are due to shutting off the ability to respond to the gelatinase biosynthesis-activating pheromone (GBAP) quorum-sensing signal. Microbiol. 2012;158(Pt 2):519-28.

74. Coburn PS, Baghdayan AS, Dolan GT, Shankar N. Horizontal transfer of virulence genes encoded on the Enterococcus faecalis pathogenicity island. Mol Microbiol. 2007;63(2):530-44.

75. Bazaka K, Crawford RJ, Nazarenko EL, Ivanova EP. Bacterial extracellular polysaccharides. Adv Experim Med Biol. 2011;715:213-26.

76. Hufnagel M, Sixel K, Hammer F, Kropec A, Sava IG, Theilacker C, et al. Detection of opsonic antibodies against Enterococcus faecalis cell wall carbohydrates in immune globulin preparations. Infection. 2014;42:749-55.

77. Lynn E, Hancock BEM, Jouko S. Enterococcal cell wall components and structures. In: Gilmore DBC MS, Yasuyoshi I, Nathan S, editors. Enterococci from commensals to leading causes of drug resistant infection, vol. 2. Boston, MA: Massachusetts Eye and Ear Infirmary; 2014.

78. Gaspar FB, Montero N, Akary E, Teixeira N, Matos R, Gonzalez-Zorn B, et al. Incongruence between the cps type 2 genotype and host-related phenotypes of an Enterococcus faecalis food isolate. Int J Food Microbiol. 2012;158(2):120-5.

79. Solheim M, Brekke MC, Snipen LG, Willems RJ, Nes IF, Brede DA. Comparative genomic analysis reveals significant enrichment of mobile genetic elements and genes encoding surface structure-proteins in hospital-associated clonal complex 2 Enterococcus faecalis. BMC Microbiol. 2011;11:3.

80. Yebra MJ, Zúñiga M, Beaufils S, Pérez-Martínez G, Deutscher J, Monedero V. Identification of a gene cluster enabling Lactobacillus casei BL23 to utilize myo-inositol. Appl Environ Microbiol. 2007;73(12):3850-8.

81. Wardal E, Gawryszewska I, Hryniewicz W, Sadowy E. Abundance and diversity of plasmid-associated genes among clinical isolates of Enterococcus faecalis. Plasmid. 2013:pii: S0147-0619X(0113)00079-00076.
82. Duerkop BA, Clements CV, Rollins D, Rodrigues JL, Hooper LV. A composite bacteriophage alters colonization by an intestinal commensal bacterium. Proc Natl Acad Sci U S A. 2012;109(43):17621-6.

83. Wagner PL, Waldor MK. Bacteriophage control of bacterial virulence. Infect Immun. 2002;70(8):3985-93.

84. Winstel V, Liang C, Sanchez-Carballo P, Steglich M, Munar M, Bröker BM, et al. Wall teichoic acid structure governs horizontal gene transfer between major bacterial pathogens. Nat Commun. 2013;4:2345.

85. Jensen LB, Garcia-Migura L, Valenzuela AJ, Lohr M, Hasman H, Aarestrup FM. A classification system for plasmids from enterococci and other Gram-positive bacteria. J Microbiol Meth. 2010;80(1):25-43.

86. Weaver KE, Kwong SM, Firth N, Francia MV. The RepA_N replicons of Gram-positive bacteria: a family of broadly distributed but narrow host range plasmids. Plasmid. 2009;61(2):94-109.

87. Werner G, Coque TM, Franz CM, Grohmann E, Hegstad K, Jensen L, et al. Antibiotic resistant enterococci-Tales of a drug resistance gene trafficker. Int J Med Microbiol. 2013;303(6-7):360-79.

88. Mikalsen T, Pedersen T, Willems R, Coque TM, Werner G, et al. Investigating the mobilome in clinically important lineages of Enterococcus faecium and Enterococcus faecalis. BMC Genomics. 2015. DOI 10.1186/s12864-015-1407-6.

89. Palmer KL, Gilmore MS. Multidrug-resistant enterococci lack CRISPR-cas. mBio. 2010;1(4):pii: e00227-00210.

90. Guiton PS, Hung CS, Hancock LE, Caparon MG, Hultgren SJ. Enterococcal biofilm formation and virulence in an optimized murine model of foreign body-associated urinary tract infections. Infect Immun. 2010;78(10):4166-75.

91. Ruiz-Garbajosa P, Canton R, Pintado V, Coque TM, Willems R, Baquero F, et al. Genetic and phenotypic differences among Enterococcus faecalis clones from intestinal colonisation and invasive disease. Clin Microbiol Infect. 2006;12(12):1193-8.

92. Hancock LE, Perego M. The Enterococcus faecalis fsr two-component system controls biofilm development through production of gelatinase. J Bacteriol. 2004;186(17):5629-39.

93. Bourgogne A, Singh KV, Fox KA, Pflughoeft K, Murray BE, Garsin DA. EbpR is important for biofilm formation by activating expression of the endocarditis and biofilm-associated pilus operon (ebpABC) of Enterococcus faecalis OG1RF. J Bacteriol. 2007;189(17):6490-3.

94. Ballering KS, Kristich CJ, Grindle SM, Oromendia A, Beattie DT, Dunny GM. Functional genomics of Enterococcus faecalis: multiple novel genetic determinants for biofilm formation in the core genome. J Bacteriol. 2009;191(8):2806-14

95. Maddox SM, Coburn PS, Shankar N, Conway T. Transcriptional Regulator PerA Influences Biofilm-Associated, Platelet Binding, and Metabolic Gene Expression in Enterococcus faecalis. PLoS One. 2012;7(3):e34398.

96. Haller C, Berthold M, Wobser D, Kropec A, Lauriola M, Schlensak C, et al. Cell-wall glycolipid mutations and their effects on virulence of $E$. faecalis in a rat model of infective endocarditis. PLoS One. 2014;9(3):e91863.

97. Rudolph B. Investigations on Enterococcus faecalis as a possible cause of amyloid arthropathy in brown laying hen, Dissertation thesis. Berlin: Free University Berlin; 2004.

98. Neumann B. Comparative proteome analysis of Enterococcus faecalis strains from clinical and food sources. Greifswald: Ernst-Moritz-Arndt-University Greifswald, Bachelor thesis; 2013.

99. Benachour A, Morin T, Hébert L, Budin-Verneuil A, Le Jeune A, Auffray Y, et al. Identification of secreted and surface proteins from Enterococcus faecalis. Can J Microbiol. 2009;55(8):967-74.

100. Bøhle LA, Riaz T, Egge-Jacobsen W, Skaugen M, Busk ØL, Eijsink VG, et al. Identification of surface proteins in Enterococcus faecalis V583. BMC Genomics. 2011;12:135.

101. Schwarz FV, Perreten V, Teuber M. Sequence of the 50-kb conjugative multiresistance plasmid pRE25 from Enterococcus faecalis RE25. Plasmid. 2001;46(3):170-87. 